\title{
The issue of gauge choice in the Landau problem and the physics of canonical and mechanical orbital angular momenta
}

\author{
M. Wakamatsu ${ }^{\text {a,b,*, Y. Kitadono }}$, P.-M. Zhang ${ }^{\text {a }}$ \\ ${ }^{a}$ Institute of Modern Physics, Chinese Academy of Sciences, \\ Lanzhou, People's Republic of China, 730000 \\ ${ }^{b}$ KEK Theory Center, Institute of Particle and Nuclear Studies, \\ High Energy Accelerator Research Organization (KEK), \\ 1-1, Oho, Tsukuba, Ibaraki 305-0801, Japan
}

\begin{abstract}
One intriguing issue in the nucleon spin decomposition problem is the existence of two types of decompositions, which are representably characterized by two different orbital angular momenta (OAMs) of quarks. The one is the mechanical OAM, while the other is the so-called gauge-invariant canonical (g.i.c.) OAM, the concept of which was introduced by Chen et al. An especially delicate quantity is the g.i.c. OAM, which must be distinguished from the ordinary (gauge-variant) canonical OAM. We find that, owing to its analytically solvable nature, the famous Landau problem offers an ideal tool to understand the difference and the physical meaning of the above three OAMs, i.e. the standard canonical OAM, g.i.c. OAM, and the mechanical OAM. We analyze these three OAMs in two different formulations of the Landau problem, first in the standard (gauge-fixed) formulation and second in the gauge-invariant (but path-dependent) formulation of DeWitt. Especially interesting is the latter formalism. It is shown that the choice of path has an intimate connection with the choice of gauge, but they are not necessarily equivalent. Then, we answer the question about what is the consequence of a particular choice of path in DeWitt's formalism. This analysis also clarifies the implication of the gauge symmetry hidden in the concept of g.i.c. OAM. Finally, we show that the finding above offers a clear understanding about the uniqueness or non-uniqueness problem of the nucleon spin decomposition, which arises from the arbitrariness in the definition of the so-called physical component of the gauge field.
\end{abstract}

Keywords: Landau problem, gauge choice, gauge-invariant formulation, path dependence, orbital angular momenta, nucleon spin decomposition

71.70.Di, 03.65.-w, 11.15.-q, 11.30.-j

\section{Introduction}

How the total nucleon spin can be decomposed into the spin and orbital angular momentum $(\mathrm{OAM})$ of quarks and gluons without conflicting with the gauge-invariance principle is a very

\footnotetext{
* corresponding author

Email address: wakamatu@post .kek.jp (M. Wakamatsu)
} 
delicate problem of quantum chromodynamics (QCD), and a lot of debates were developed for a considerable time. (See [1],[2],[3] for review.) A consensus now is that there are two different types of complete decomposition of the nucleon spin, which are respectively called the canonical type decomposition and the mechanical type one [4],[5]. Unfortunately, there still remains different opinions on the proper physical interpretation of the two decompositions of the nucleon spin. Since these two decompositions are representably characterized by the canonical OAM and the mechanical OAM of quarks, the judgment on the merits and demerits of the two nucleon spin decomposition cannot be delivered unless we do understand the difference and the physical meanings of these two OAMs correctly.

To understand the difference of these two OAMs in a clearest fashion, the familiar Landau problem turns out to offer an ideal testing ground, because of its analytically solvable nature. The Landau problem is a quantum mechanical problem, which describes the motion of an electron or any charged particle in a uniform magnetic field [6], [7]. Since the Hamiltonian of the Landau problem contains the vector potential not the magnetic field itself, it has a freedom of gauge choice. In the standard treatment of the Landau problem, one first fixes a gauge and then solves the quantum mechanical Schrödinger equation. Typical gauge choices are the two types of Landau gauge and the symmetric gauge. Less popular is a gauge-invariant formulation of the Landau problem, in which the Schrödinger equation can be solved without assuming any particular form of vector potential, which means that the problem can be solved without fixing gauge.

The purpose of the present study is to clearly understand the physical meaning of the two OAMs, i.e. the canonical OAM and the mechanical OAM of the electron, by making full use of the analytically solvable nature of the Landau problem. What plays an important role in this analysis is the existence of another OAM called the pseudo angular momentum in the literature [8],[9]. (A similar quantity called the pseudo momentum was already discussed in [10].) A unique feature of this quantity is that it is gauge-invariant, and reduces to the canonical OAM in a suitable gauge. Because of this property, the pseudo OAM is very much resembling the quantity called the gauge-invariant-canonical (g.i.c.) OAM, the concept of which was introduced by Chen et al. in the discussion of the nucleon spin decomposition problem as well as the nucleon momentum decomposition problem [11],[12]. (In the following, we mainly use the latter name instead of the terminology pseudo angular momentum.) In view of this situation, we are faced with three types of OAM. The first is the ordinary canonical OAM, which is usually believed to be a gauge-variant quantity. The second is the g.i.c. OAM. The last is the manifestly gaugeinvariant mechanical OAM. We aim at clarifying the physical meaning of these three OAMs, by paying special attention to their gauge-dependence or gauge-independence. We shall carry out this analysis in both of the standard (gauge-fixed) formulation of the Landau problem and also in the gauge-invariant formulation of the Landau problem. It turns out that this parallel analysis is particularly useful not only for clarifying the physical meaning of the above three OAMs but also for getting deeper understanding of the delicacy hidden in the gauge choices in the Landau problem as well as in the nucleon spin decomposition problem.

The plan of the paper is as follows. In section2 a theoretical analysis of the above-mentioned three OAMs is carried out within the framework of the standard formulation of the Landau problem. We first show that the standard eigen-functions of the Landau Hamiltonian in the Landau gauge is not connected with the standard eigen-functions of the symmetric gauge by a simple gauge transformation. And then, we derive the correct relation between these two eigen-functions in the two different gauges. Next in section 3, the same three OAMs are analyzed within the framework of the gauge-invariant formulation of the Landau problem. The delicacy of the gauge choice in the Landau problem will become clear by combining the analysis in this section and 
that in the previous section. In section 4 we discuss in some detail the similarity between the concept of pseudo momentum and pseudo OAM and that of g.i.c. momentum and g.i.c. OAM of Chen et al. This consideration is useful for unraveling the delicacy hidden in the idea of g.i.c. momentum and g.i.c. OAM. Next in section 5, we make some important remarks on the issue of gauge choice in the nucleon spin decomposition problem. Putting all the knowledge obtained so far in force from our general viewpoint of the gauge-invariant but path-dependent formulation of gauge theory, we will clarify what is really meant by the idea of the physical component of the gauge field, which was introduced by Chen et al. Finally in section 6 , we summarize what we have found and make some concluding remarks.

\section{Canonical and mechanical orbital angular momenta in the standard formulation of the Landau problem}

The Landau problem is a very fundamental quantum mechanical problem, which describes the motion of an electron, or any charged particle, under the influence of uniform magnetic field. In the following, the direction of the magnetic field is taken in the $z$-direction, and the mass and the charge of the electron are denoted as $m_{e}$ and $-e$ with $e>0$. (The natural unit $c=\hbar=1$ is used throughout the paper.)

The Hamiltonian of the system is represented as

$$
H=\frac{\Pi^{2}}{2 m_{e}},
$$

in terms of the so-called mechanical (or kinetic) momentum $\boldsymbol{\Pi}$ defined as

$$
\boldsymbol{\Pi}=\boldsymbol{p}+e \boldsymbol{A},
$$

with $\boldsymbol{A}$ being the vector potential giving the magnetic field through the relation $\nabla \times \boldsymbol{A}=\boldsymbol{B}=B \boldsymbol{e}_{z}$.

As is well-known, the choice of vector potential, which gives the same magnetic field, is not unique. We say that there is a freedom of gauge choices. The most popular gauge choices are the following three :

$$
\begin{aligned}
\text { 1st Landau gauge } & : A=\boldsymbol{A}_{L_{1}}=-B y \boldsymbol{e}_{x}, \\
\text { 2nd Landau gauge } & : \quad \boldsymbol{A}=\boldsymbol{A}_{L_{2}}=+B x \boldsymbol{e}_{y}, \\
\text { symmetric gauge } & : \quad \boldsymbol{A}=\boldsymbol{A}_{S}=-\frac{1}{2} B y \boldsymbol{e}_{x}+\frac{1}{2} B x \boldsymbol{e}_{y} .
\end{aligned}
$$

The two Landau gauges can be treated almost in the same manner, so that in this section we consider the 2 nd Landau gauge and call it simply the Landau gauge.

Our primary concern in the present paper is to unravel the physical meaning of the two types of orbital angular momentum (OAM), i.e. the canonical OAM,

$$
L_{c a n} \equiv(\boldsymbol{r} \times \boldsymbol{p})_{z}=-i\left(x \frac{\partial}{\partial y}-y \frac{\partial}{\partial x}\right),
$$

and the mechanical OAM,

$$
L_{\text {mech }} \equiv(\boldsymbol{r} \times \boldsymbol{\Pi})_{z}=(\boldsymbol{r} \times \boldsymbol{p})_{z}+e(\boldsymbol{r} \times \boldsymbol{A})_{z} .
$$


They are related as

$$
L_{c a n}=L_{m e c h}+L_{p o t},
$$

where

$$
L_{p o t} \equiv-e(\boldsymbol{r} \times \boldsymbol{A})_{z}=-e\left(x A_{y}-y A_{x}\right) .
$$

Here, $L_{p o t}$ is basically the quantity, which was called in [4],[5] the potential angular momentum. (To be more rigorous, the potential angular momentum introduced in [4],[5] is defined by $L_{p o t}=-e\left(\boldsymbol{r} \times \boldsymbol{A}_{\perp}\right)_{z}$ with $\boldsymbol{A}_{\perp}$ being the transverse component of $\boldsymbol{A}$, and it is gauge-invariant under the residual gauge transformation within the Coulomb gauge.) The potential angular momentum defined by (9) is generally gauge-dependent and takes the following form according to the choices of gauge :

$$
\begin{array}{rll}
\text { symmetric gauge } & : & L_{p o t}=-\frac{1}{2} e B\left(x^{2}+y^{2}\right), \\
\text { Landau gauge } & : & L_{p o t}=-e B x^{2} .
\end{array}
$$

At this stage, it is useful to recall the known solution to the Landau problem, i.e. the eigenvalues and the eigen-functions of the Landau Hamiltonian $H$. The standardly-known eigenfunctions of $H$ in the Landau gauge are given as [6], [7]

$$
\Psi_{n, k_{y}}^{(L)}(x, y)=\frac{1}{\sqrt{2 \pi}} e^{i k_{y} y} N_{n} H_{n}\left(\frac{x-x_{0}}{l_{B}}\right) e^{-\frac{\left(x-x_{0}\right)^{2}}{2 l_{B}^{2}}},
$$

where $H_{n}(x)$ are the Hermite polynomials, while the other constants are defined by

$$
N_{n}=\left(\frac{1}{\sqrt{\pi} 2^{n} n ! l_{B}}\right)^{1 / 2},
$$

and

$$
x_{0}=-l_{B}^{2} k_{y} \text { with } l_{B}^{2}=\frac{1}{e B} .
$$

The quantity $x_{0}$ is sometimes called the guiding center, while $l_{B}$ is called the magnetic length. The above wave functions are the simultaneous eigen-functions of the canonical momentum operator $p_{y}$ and the Landau Hamiltonian $H$ :

$$
\begin{aligned}
p_{y} \Psi_{n, k_{y}}^{(L)}(x, y) & =k_{y} \Psi_{n, k_{y}}^{(L)}(x, y), \\
H \Psi_{n, k_{y}}^{(L)}(x, y) & =\left(n+\frac{1}{2}\right) \omega \Psi_{n, k_{y}}^{(L)}(x, y) \quad(n=0,1,2, \cdots),
\end{aligned}
$$

where $n=0,1,2, \cdots$, and $\omega=\frac{e B}{m_{e}}$. Note that the eigen-energies depend only on the so-called Landau quantum number $n$, and they do not depend on the quantum number $k_{y}$.

On the other hand, the standard eigen-functions in the symmetric gauge are given as [13], [14]

$$
\Psi_{n, m}^{(S)}(x, y)=\frac{1}{\sqrt{2 \pi}} e^{i m \phi} N_{n, m}\left(\frac{r^{2}}{2 l_{B}^{2}}\right)^{|m| / 2} e^{-\frac{r^{2}}{4 l_{B}^{2}}} L_{n-\frac{|m|+m}{2}}^{|m|}\left(\frac{r^{2}}{2 l_{B}^{2}}\right)
$$


with $m$ being an integer satisfying the condition $m \leq n, L_{n}^{m}(x)$ is the associated Laguerre polynomial, while

$$
N_{n, m}=(-1)^{n+\frac{|m|+m}{2}} \frac{1}{l_{B}} \sqrt{\frac{\left(n-\frac{|m|+m}{2}\right)}{\left(n+\frac{|m|-m}{2}\right)}} .
$$

They are the simultaneous eigen-functions of the canonical OAM operator $L_{c a n}$ and the Landau Hamiltonian $H$ :

$$
\begin{aligned}
L_{c a n} \Psi_{n, m}^{(S)}(x, y) & =m \Psi_{n, m}^{(S)}(x, y), \\
H \Psi_{n, m}^{(S)}(x, y) & =\left(n+\frac{1}{2}\right) \omega \Psi_{n, m}^{(S)}(x, y),
\end{aligned}
$$

Here, we are interested in the expectation values of the canonical and mechanical OAM operators in the above two gauges. The answer to this question is well known in the symmetric gauge. One can easily confirm that

$$
\begin{aligned}
& \left\langle L_{c a n}\right\rangle=\left\langle\Psi_{n, m}^{(S)}(x, y)\left|L_{c a n}\right| \Psi_{n, m}^{(S)}(x, y)\right\rangle=m, \\
& \left\langle L_{p o t}\right\rangle=\left\langle\Psi_{n, m}^{(S)}(x, y)\left|-\frac{1}{2} e B r^{2}\right| \Psi_{n, m}^{(S)}(x, y)\right\rangle=-(2 n+1-m),
\end{aligned}
$$

which in turn gives

$$
\left\langle L_{m e c h}\right\rangle=\left\langle L_{c a n}\right\rangle-\left\langle L_{p o t}\right\rangle=m+(2 n+1-m)=2 n+1 .
$$

(We point out that the overall sign of the expectation value of the mechanical OAM is opposite to that in [15]. This is because we consider here the motion of the electron with negative charge in contrast to [15], in which the motion of a particle with positive charge is studied. Note that the cyclotron motion of a particle with negative charge is counterclockwise in the $x-y$ plane, so that its mechanical angular momentum should be positive.)

Remarkably, the expectation value of the mechanical OAM depends only on the Landau quantum number $n$, and it does not depends on the eigen-value $m$ of the canonical OAM. We also recall that the expectation value of the mechanical OAM is just proportional to the eigen-energies of the Landau level, or the expectation value of the Landau Hamiltonian :

$$
E_{n}=\left(n+\frac{1}{2}\right) \omega=\frac{e B}{2 m_{e}}\left\langle\Psi_{n, m}^{(S)}\left|L_{\text {mech }}\right| \Psi_{n, m}^{(S)}\right\rangle .
$$

This is consistent with the known fact that what describes the physical cyclotron motion is the mechanical OAM not the canonical one.

One might naturally ask the following question. What are expectation values of the two angular momentum operators in the Landau gauge ? Since the mechanical OAM is generally believed to be a gauge-invariant operator, one might at least anticipate that the expectation value of the mechanical OAM operator is independent of the gauge choice, i.e. it is the same in both the symmetric and Landau gauges. The problem is more subtle than this naive expectation. To see it, imagine that we want to evaluate the expectation value of the canonical OAM operator in the Landau gauge, or more precisely the expectation value between the standard eigen-states in the Landau gauge,

$$
\left\langle L_{c a n}\right\rangle \equiv\left\langle\Psi_{n, k_{y}}^{(L)}|-i \underbrace{}_{5}\left(x \frac{\partial}{\partial y}-y \frac{\partial}{\partial x}\right)| \Psi_{n, k_{y}}^{(L)}\right\rangle .
$$


First, this expression must be taken with care, because the plane-wave in the $y$-direction is not normalizable. To make the expression finite, the box normalization was adopted in [15], which means the replacement

$$
\frac{1}{\sqrt{2 \pi}} e^{i k_{y} y} \rightarrow \frac{1}{\sqrt{L}} e^{i k_{y} y} \text { with } \quad-\frac{L}{2} \leq y \leq \frac{L}{2} .
$$

With this replacement, the expectation value of the canonical OAM operator becomes

$$
\left\langle L_{c a n}\right\rangle=-\frac{k_{y}^{2}}{e B} .
$$

With the same box normalization, the expectation value of the potential OAM operator is shown to be

$$
\left\langle L_{p o t}\right\rangle \equiv\left\langle\Psi_{n, k_{y}}^{(L)}\left|-e B x^{2}\right| \Psi_{n, k_{y}}^{(L)}\right\rangle=-\left\{\left(n+\frac{1}{2}\right)+\frac{k_{y}^{2}}{e B}\right\} .
$$

(There is a factor of two mistake in Eq.(59) of [15], which gives $2 n+1$ instead of $n+\frac{1}{2}$ in the above equation. This led to an erroneous conclusion that the expectation value of the mechanical OAM operator in the Landau gauge, or more precisely in the expectation value in the standard eigen-functions in the Landau gauge, is the same as that in the symmetric gauge. See below for more detail.) The expectation value of the mechanical OAM operator can easily be obtained from the above two relations :

$$
\left\langle L_{m e c h}\right\rangle=\left\langle L_{c a n}\right\rangle-\left\langle L_{p o t}\right\rangle=n+\frac{1}{2} .
$$

We find that the $k_{y}^{2}$ dependent terms in the expectation values of the canonical OAM and potential OAM terms cancel exactly. However, the expectation value of the mechanical OAM in the Landau gauge turns out to be half of the corresponding expectation value in the symmetric gauge. At first glance, this result appears to contradict the standard belief that the mechanical OAM operator is a gauge-invariant quantity. However, it would be more legitimate to say that the $k_{y}^{2}$ dependent terms, contained in both of (27) and (28), do not have clear physical meaning at least in its present form. This is due to the fact that, for the eigen-functions in the Landau gauge, $y$-coordinate of the electron is totally uncertain. After all, we conclude that the concept of orbital angular momentum does not have well-defined physical meaning within the standard eigen-functions in the Landau gauge.

The above analysis shows that the issue of the gauge-invariance of the mechanical OAM in the Landau problem should be investigated more carefully. It is a widely-known fact that the two gauge choices, i.e. the symmetric gauge and the Landau gauge, are related by the gauge transformation, $\boldsymbol{A}_{L}=\boldsymbol{A}_{S}+\nabla \chi$ with $\chi=\frac{1}{2} B x y$. To understand the true meaning of this gauge transformation, we first briefly review the consequence of gauge-invariance in quantum mechanics. In quantum mechanics, the gauge transformation

$$
\boldsymbol{A}^{\prime}=\boldsymbol{A}+\nabla \chi
$$

must be supplemented with the phase transformation of the charged particle wave function

$$
\psi^{\prime}(\boldsymbol{r})=e^{i q \chi(\boldsymbol{r})} \psi(\boldsymbol{r}),
$$


such that the identity

$$
\left(\hat{\boldsymbol{p}}-q \boldsymbol{A}^{\prime}(\boldsymbol{r})\right) \psi^{\prime}(\boldsymbol{r})=e^{i q \chi(\boldsymbol{r})}(\hat{\boldsymbol{p}}-q \boldsymbol{A}(\boldsymbol{r})) \psi(\boldsymbol{r}),
$$

holds. The standard statement is that observables of the type

$$
\left\langle\psi_{f}^{\prime}(\boldsymbol{r})\left|O_{1}(\boldsymbol{r})\right| \psi_{i}^{\prime}(\boldsymbol{r})\right\rangle=\left\langle\psi_{f}(\boldsymbol{r})\left|O_{1}(\boldsymbol{r})\right| \psi_{i}(\boldsymbol{r})\right\rangle,
$$

and also of the type

$$
\left\langle\psi_{f}^{\prime}(\boldsymbol{r})\left|O_{2}\left(\hat{\boldsymbol{p}}-q \boldsymbol{A}^{\prime}\right)\right| \psi_{i}^{\prime}(\boldsymbol{r})\right\rangle=\left\langle\psi_{f}(\boldsymbol{r})\left|O_{2}(\hat{\boldsymbol{p}}-q \boldsymbol{A})\right| \psi_{i}(\boldsymbol{r})\right\rangle,
$$

are unchanged by the gauge transformations, i.e. they are gauge-invariant.

The coordinate operator $\hat{\boldsymbol{r}}$ is the simplest example of the first type of observables. The mechanical momentum $\hat{\boldsymbol{p}}-q \boldsymbol{A}$ as well as the mechanical orbital angular momentum $\boldsymbol{r} \times(\hat{\boldsymbol{p}}-q \boldsymbol{A})$ are special examples of the 2 nd types of operator. (Aside from the above few paragraphs, the quantum operators $\hat{\boldsymbol{r}}$ and $\hat{\boldsymbol{p}}$ are simply denoted as $\boldsymbol{r}$ and $\boldsymbol{p}$, since no confusion is expected to occur.) From the general statement above, one might naively anticipate that the following relation would hold

$$
\left\langle\Psi^{(L)}\left|L_{\text {mech }}^{(L)}\right| \Psi^{(L)}\right\rangle=\left\langle\Psi^{(S)}\left|L_{\text {mech }}^{(S)}\right| \Psi^{(S)}\right\rangle .
$$

Here, $L_{\text {mech }}^{(S)}=\left[\boldsymbol{r} \times\left(\boldsymbol{p}+e \boldsymbol{A}_{S}\right)\right]_{z}$ is the mechanical OAM operator in the symmetric gauge, while $\left|\Psi^{(S)}\right\rangle$ are the eigen-states of the Landau Hamiltonian in the same gauge. On the other hand, $L_{\text {mech }}^{(L)}=\left[\boldsymbol{r} \times\left(\boldsymbol{p}+\boldsymbol{e} \boldsymbol{A}_{L}\right)\right]_{z}$ is the mechanical OAM operator in the Landau gauge, while $\left|\Psi^{(L)}\right\rangle$ are the eigen-states of the Landau Hamiltonian in the Landau gauge. These quantities are supposed to be related by the unitary (gauge) transformation $U=e^{-\frac{1}{2} i e B x y}$ as

$$
\left|\Psi^{(L)}\right\rangle=U\left|\Psi^{(S)}\right\rangle,
$$

and

$$
L_{\text {mech }}^{(L)}=U L_{\text {mech }}^{(S)} U^{\dagger} .
$$

However, it turns out that there is an oversight in the above naive reasoning. This is due to the well-known degeneracy of the eigen-states of the Landau Hamiltonian [16]. An important point is that the eigen-states of the Landau Hamiltonian with a given Landau quantum number $n$ has infinitely many degeneracies in both of the symmetric gauge and the Landau gauge. Because of this degeneracy, it happens that the standard wave functions in the Landau gauge are not connected with those in the symmetric gauge by a simple gauge transformation.

To understand this complexity in more concrete and clearer way, let us consider the operation of the gauge transformation matrix $U$ on the eigen-states $\left|\Psi_{n, m}^{(S)}\right\rangle$ in the symmetric gauge, which are characterized by the two quantum numbers, i.e. the Landau quantum number $n$ and the eigenvalue $m$ of the canonical OAM operator. With use of the completeness relation for the eigen-states $\left|\Psi_{n, k_{y}}^{(L)}\right\rangle$ in the Landau gauge, we generally have

$$
U\left|\Psi_{n, m}^{(S)}\right\rangle=\sum_{n^{\prime}} \int d k_{y}\left|\Psi_{n^{\prime}, k_{y}}^{(L)}\right\rangle\left\langle\Psi_{n^{\prime}, k_{y}}^{(L)}|U| \Psi_{n, m}^{(S)}\right\rangle,
$$

where

$$
\left\langle\Psi_{n^{\prime}, k_{y}}^{(L)}|U| \Psi_{n, m}^{(S)}\right\rangle \equiv \int d x d y \Psi_{n^{\prime}, k_{y}}^{(L)}(x, y) e^{-i \frac{1}{2} e B x y} \Psi_{n, m}^{(S)}(x, y) .
$$


In Appendix A, we show that the above gauge transformation matrix is diagonal in the Landau quantum number, i.e.

$$
\left\langle\Psi_{n^{\prime}, k_{y}}^{(L)}|U| \Psi_{n, m}^{(S)}\right\rangle=\delta_{n^{\prime}, n}\left\langle\Psi_{n, k_{y}}^{(L)}|U| \Psi_{n, m}^{(S)}\right\rangle .
$$

Actually, this is an anticipated fact, since the gauge transformation does not change the Landau energy, which is characterized by the quantum number $n$.

Since the above gauge transformation matrix is a function of $k_{y}$ or $x_{0}=-l_{B}^{2} k_{y}$, we denote it as $U_{n, m}\left(x_{0}\right)$, i.e.

$$
\left\langle\Psi_{n, k_{y}}^{(L)}|U| \Psi_{n, m}^{(S)}\right\rangle \equiv U_{n, m}\left(x_{0}\right)
$$

Thus Eq. 38 can be written as

$$
U\left|\Psi_{n, m}^{(S)}\right\rangle=\int d k_{y} U_{n, m}\left(x_{0}\right)\left|\Psi_{n, k_{y}}^{(L)}\right\rangle .
$$

This relation shows what is generated by the action of the gauge transformation operator $U$ on the eigen-states $\left|\Psi_{n, m}^{(S)}\right\rangle$ in the symmetric gauge is a superposition of the eigen-states $\left|\Psi_{n, k_{y}}^{(L)}\right\rangle$ in the Landau gauge with respect to the variable $k_{y}$ or $x_{0}$. The above transformation matrix $U_{n, m}\left(x_{0}\right)$ corresponds to the matrix $R_{n, m}\left(x_{0}\right)$ in the paper by Haugset et al. [17], although this fact is not so transparent, since their treatment is based on a sort of gauge-invariant formulation of the Landau problem. In our present treatment, which is based on the standard (gauge-fixed) formulation of the Landau problem, the weight function $U_{n, m}\left(x_{0}\right)$ appearing in (42) is just the matrix element of the gauge transformation operator $U$ sandwiched with the standard eigen-states in the Landau and symmetric gauges.

The calculation of the weight function $U_{n, m}\left(x_{0}\right)$ is elementary but a little involved, so that the detailed derivation is shown in Appendix A. The answer is given as

$$
U_{n, m}\left(x_{0}\right)=C_{n, m} H_{n-m}\left(\frac{x_{0}}{l_{B}}\right) e^{-\frac{x_{0}^{2}}{2 l_{B}^{2}}},
$$

where $H_{n}(x)$ is the standard Hermite polynomial, and

$$
C_{n, m}=l_{B}\left(\frac{1}{\sqrt{\pi} 2^{n-m}(n-m) ! l_{B}}\right)^{1 / 2} .
$$

Now, inserting (43) into the r.h.s. of (42), we can show that the following relation holds

$$
\int d k_{y} U_{n, m}\left(x_{0}\right) \Psi_{n, k_{y}}^{(L)}(x, y)=e^{-i \frac{1}{2} e B x y} \Psi_{n, m}^{(S)}(x, y)
$$

The proof of this relation is given in Appendix B.

It is convenient to define the 1.h.s. of the above equation newly as $\Psi_{n, m}^{(L)}(x, y)$, i.e.

$$
\Psi_{n, m}^{(L)}(x, y) \equiv \int d k_{y} U_{n, m}\left(x_{0}\right) \Psi_{n, k_{y}}^{(L)}(x, y) .
$$

Then, the relation that we have found can be expressed as

$$
\Psi_{n, m}^{(L)}(x, y)=e^{-i \frac{1}{2} e B x y} \Psi_{n, m}^{(S)}(x, y) .
$$


We may now say that the 1.h.s. of the above equation gives wave functions in the Landau gauge, which is obtained from the eigen-functions in the symmetric gauge by the simple gauge transformation with the gauge function $\chi=\frac{1}{2} B x y$. However, the important point is that they are not the standard eigen-functions in the Landau gauge but their particular superpositions.

We are now ready to evaluate the expectation values of the orbital angular momentum operators

$$
\begin{aligned}
L_{c a n} & =-i\left(x \frac{\partial}{\partial y}-y \frac{\partial}{\partial x}\right), \\
L_{p o t} & =-e B x^{2} \\
L_{m e c h} & =L_{c a n}-L_{p o t},
\end{aligned}
$$

between the superposed eigenstates $\Psi_{n, m}^{(L)}(x, y)$ in the Landau gauge. With use of the relation 477, the expectation values of the canonical OAM and the potential OAM can be easily obtained as

$$
\left\langle\Psi_{n, m}^{(L)}\left|L_{c a n}\right| \Psi_{n, m}^{(L)}\right\rangle=\left\langle\Psi_{n, m}^{(L)}\left|-i\left(x \frac{\partial}{\partial y}-y \frac{\partial}{\partial y}\right)\right| \Psi_{n, m}^{(L)}\right\rangle=m,
$$

and

$$
\left\langle\Psi_{n, m}^{(L)}\left|L_{p o t}\right| \Psi_{n, m}^{(L)}\right\rangle=\left\langle\Psi_{n, m}^{(L)}\left|-e B x^{2}\right| \Psi_{n, m}^{(L)}\right\rangle=-(2 n+1-m)
$$

Combining these, we finally get

$$
\begin{aligned}
\left\langle\Psi_{n, m}^{(L)}\left|L_{m e c h}\right| \Psi_{n, m}^{(L)}\right\rangle & =\left\langle\Psi_{n, m}^{(L)}\left|L_{c a n}\right| \Psi_{n, m}^{(L)}\right\rangle-\left\langle\Psi_{n, m}^{(L)}\left|L_{p o t}\right| \Psi_{n, m}^{(L)}\right\rangle \\
& =m+(2 n+1-m)=(2 n+1) .
\end{aligned}
$$

This precisely coincides with the expectation value of the mechanical OAM operator evaluated in the symmetric gauge. We have thus confirmed that the mechanical OAM is in fact a gaugeinvariant quantity.

\section{Canonical and mechanical orbital angular momenta in the gauge-invariant formulation of the Landau problem}

First, we briefly review the general framework of the gauge-invariant formulation of quantum electrodynamics (QED) a la DeWitt [18] and Mandelstam [19]. (See also [20].) According to DeWitt, once an appropriate set of electron and photon fields $\left(\psi(x), A_{\mu}(x)\right)$ is given, the gaugeinvariant set of the electron and photon fields $\left(\tilde{\psi}(x), \tilde{A}_{\mu}(x)\right)$ can be constructed as

$$
\begin{aligned}
\tilde{\psi}(x) & \equiv e^{i e \Lambda(x)} \psi(x), \\
\tilde{A}_{\mu}(x) & \equiv A_{\mu}(x)-\partial_{\mu} \Lambda(x),
\end{aligned}
$$

by introducing the function

$$
\Lambda(x)=\int_{0}^{1} A_{\sigma}(z) \frac{\partial z^{\sigma}}{\partial \xi} d \xi
$$

where $z^{\mu}(x, \xi)$ represents a point on the line connecting an appropriate starting point $x_{0}$ (the reference point) and the point $x$ in the 4-dimensional Minkowski space, with $\xi$ being a parameter specifying the path with the following boundary conditions :

$$
\begin{aligned}
& z^{\mu}(x, 1)=x^{\mu}, \quad \text { and } \quad z^{\mu}(x, 0)=x_{0}^{\mu} \text {, } \\
& \left.\frac{\partial z^{\mu}}{\partial x^{\lambda}}\right|_{\xi=1}=\delta_{\lambda}^{\mu}, \quad \text { and }\left.\quad \frac{\partial z^{\mu}}{\partial x^{\lambda}}\right|_{\xi=0}=0 \text {. }
\end{aligned}
$$


Under an arbitrary gauge transformation for the electron and photon fields given by

$$
\begin{aligned}
\psi(x) & \rightarrow e^{-i e\left[\omega(x)-\omega\left(x_{0}\right)\right]} \psi(x), \\
A_{\mu}(x) & \rightarrow A_{\mu}(x)+\partial_{\mu}\left[\omega(x)-\omega\left(x_{0}\right)\right]=A_{\mu}(x)+\partial_{\mu} \omega(x),
\end{aligned}
$$

the function $\Lambda(x)$ transforms as

$$
\begin{aligned}
\Lambda(x) & \rightarrow \int_{0}^{1}\left(A_{\sigma}(z)+\partial_{\sigma} \omega(z)\right) \frac{\partial z^{\sigma}}{\partial \xi} d \xi \\
& =\int_{0}^{1} A_{\sigma}(z) \frac{\partial z^{\sigma}}{\partial \xi} d \xi+\int_{0}^{1} \frac{\partial \omega(z)}{\partial \xi} d \xi=\Lambda(x)+\left[\omega(x)-\omega\left(x_{0}\right)\right]
\end{aligned}
$$

One may notice that our gauge transformation rule (58) is somewhat unusual in the sense that it depends on the reference point $x_{0}$ in addition to the point $x$ where the field is evaluated. In the original formulation of DeWitt, this peculiarity is hidden in some sense, since the reference point is taken to be space-time infinity. Our formulation below needs to take this reference point to be some point in a finite region. As a consequence, the gauge trans,formation rule (58) is required for that the gauge-invariant electron and photon fields defined by (53) and (54) are in fact gaugeinvariant, as explicitly demonstrated below. Formally, the transformation rule (58) amounts to combining a gauge transformation with a global phase transformation. Especially, it just reduces to an identity at the reference point $x_{0}$. As we shall see, the above gauge transformation rule does not cause any practical problem in our following analysis.

In any case, under the above gauge transformation, the field $\tilde{\psi}(x)$ transforms as

$$
\begin{aligned}
\tilde{\psi}(x) & \rightarrow e^{i e\left[\Lambda(x)+\left(\omega(x)-\omega\left(x_{0}\right)\right)\right]} e^{-i e\left(\omega(x)-\omega\left(x_{0}\right)\right)} \psi(x) \\
& =e^{i e \Lambda(x)} \psi(x)=\tilde{\psi}(x),
\end{aligned}
$$

i.e., the new electron wave function $\tilde{\psi}(x)$ is gauge-invariant. The gauge-invariance of $\tilde{A}_{\mu}(x)$ can also be readily verified. For the sake of completeness, we reproduce the proof. The proof goes as follows :

$$
\begin{aligned}
\tilde{A}_{\mu}(x)= & A_{\mu}(x)-\partial_{\mu} \Lambda(x) \\
= & A_{\mu}-\partial_{\mu} \int_{0}^{1} A_{\sigma}(z) \frac{\partial z^{\sigma}}{\partial \xi} d \xi \\
= & A_{\mu}-\int_{0}^{1} \partial_{v} A_{\sigma}(z) \frac{\partial z^{\nu}}{\partial x^{\mu}} \frac{\partial z^{\sigma}}{\partial \xi} d \xi-\int_{0}^{1} A_{\sigma}(z) \frac{\partial}{\partial \xi}\left(\frac{\partial z^{\sigma}}{\partial x^{\mu}}\right) d \xi \\
= & A_{\mu}-\int_{0}^{1} \partial_{v} A_{\sigma}(z) \frac{\partial z^{\nu}}{\partial x^{\mu}} \frac{\partial z^{\sigma}}{\partial \xi} d \xi \\
& \quad+\int_{0}^{1} \partial_{v} A_{\sigma}(z) \frac{\partial z^{\nu}}{\partial \xi} \frac{\partial z^{\sigma}}{\partial x^{\mu}} d \xi-\left.A_{\sigma}(z) \frac{\partial z^{\sigma}}{\partial x^{\mu}}\right|_{\xi=0} ^{\xi=1} \\
= & A_{\mu}-\int_{0}^{1} \partial_{v} A_{\sigma}(z) \frac{\partial z^{\nu}}{\partial x^{\mu}} \frac{\partial z^{\sigma}}{\partial \xi} d \xi \\
& \quad+\int_{0}^{1} \partial_{v} A_{\sigma}(z) \frac{\partial z^{\nu}}{\partial \xi} \frac{\partial z^{\sigma}}{\partial x^{\mu}} d \xi-A_{\sigma}(x) \delta_{\mu}{ }^{\sigma} \\
= & -\int_{0}^{1}\left(\partial_{v} A_{\sigma}-\partial_{\sigma} A_{v}\right) \frac{\partial z^{v}}{\partial x^{\mu}} \frac{\partial z^{\sigma}}{\partial \xi} d \xi . \\
10 &
\end{aligned}
$$


We thus find the key expression

$$
\tilde{A}_{\mu}(x)=-\int_{0}^{1} F_{v \sigma}(z) \frac{\partial z^{\nu}}{\partial x^{\mu}} \frac{\partial z^{\sigma}}{\partial \xi} d \xi .
$$

Since the r.h.s. of the above relation is expressed only in terms of gauge-invariant field-strength tensor, the gauge-invariance of $\tilde{A}_{\mu}(x)$ is self-evident. This is the essence of the gauge-invariant formulation of QED by DeWitt. Here is a catch, however. Although the r.h.s. of (63) is certainly gauge-invariant, it generally depends on the path connecting the reference point $x_{0}$ and the point $x$. (This observation is very important, because there are in principle infinitely many paths connecting the two space-time points $x_{0}$ and $x$.)

We are now ready to apply the gauge-invariant (but path-dependent) formulation of DeWitt to the Landau problem. Since the Landau problem is a non-relativistic and stationary quantum mechanical problem in two spatial dimension, we can introduce the gauge-invariant electron wave function $\tilde{\Psi}(x, y)$ by

$$
\tilde{\Psi}(x, y) \equiv \tilde{\Psi}^{(C)}(x, y)=e^{i e \Lambda(x, y)} \Psi(x, y),
$$

with the path-dependent phase factor,

$$
\Lambda(x, y)=\int_{C} A(x) \cdot d x,
$$

where $C$ is a path connecting some reference point $\left(x_{0}, y_{0}\right)$ and the point $(x, y)$ in the twodimensional plane. (We recall that the electron charge is $-e$ with $e>0$ in our notation). Alternatively, we can express the original electron wave function $\Psi(x, y)$ in terms of the gauge-invariant electron wave function $\tilde{\Psi}^{(C)}(x, y)$ as

$$
\Psi(x, y)=U(C) \tilde{\Psi}^{(C)}(x, y),
$$

with

$$
U(C)=e^{-i e \int_{C} A(x) \cdot d x} .
$$

In the following, two different types of path choice will be considered, separately in two subsections.

\section{1. polygonal line paths in rectangular coordinate}

Two simple choices of path connecting $\left(x_{0}, y_{0}\right)=(0,0)$ and $(x, y)$ in the $x-y$ plane are illustrated in Fig 1. They are made up of line segments parallel to either of the two rectangular coordinate axes :

$$
\begin{aligned}
& C_{1}:(0,0) \rightarrow(x, 0) \rightarrow(x, y), \\
& C_{2}:(0,0) \rightarrow(0, y) \rightarrow(x, y) .
\end{aligned}
$$

Selecting the path $C_{1}$, the relation between the standard electron wave function $\Psi(x, y)$ and the gauge-invariant (but path-dependent) electron wave function $\tilde{\Psi}^{\left(C_{1}\right)}(x, y)$ are given as

$$
\Psi(x, y)=U_{1} \tilde{\Psi}^{\left(C_{1}\right)}(x, y),
$$

with

$$
U_{1} \equiv e^{-i e \int_{C_{1}} A(x) \cdot d x}=e^{-i e\left\{\int_{0}^{x} A_{x}\left(x^{\prime}, 0\right) d x^{\prime}+\int_{0}^{y} A_{y}\left(x, y^{\prime}\right) d y^{\prime}\right\}} .
$$




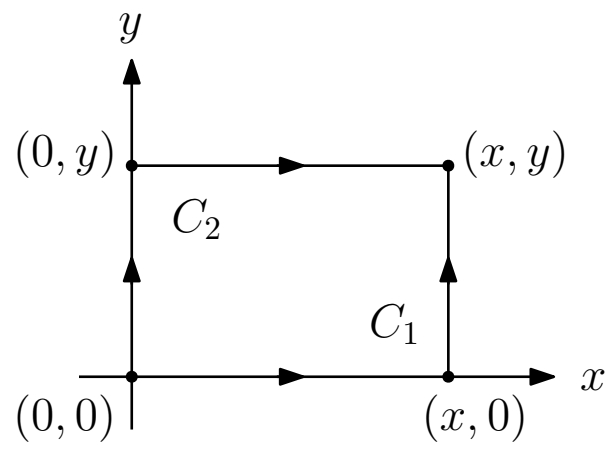

Figure 1: The two polygonal line paths $C_{1}$ and $C_{2}$ defined in the rectangular coordinate system.

On the other hand, with choice of the path $C_{2}$, we have

$$
\Psi(x, y)=U_{2} \tilde{\Psi}^{\left(C_{2}\right)}(x, y),
$$

with

$$
U_{2} \equiv e^{-i e \int_{C_{2}} \boldsymbol{A}(\boldsymbol{x}) \cdot d \boldsymbol{x}}=e^{-i e\left\{\int_{0}^{y} A_{y}\left(0, y^{\prime}\right) d y^{\prime}+\int_{0}^{x} A_{y}\left(x^{\prime}, y\right) d x^{\prime}\right\}} .
$$

There is a nontrivial relation between the two operators $U_{1}$ and $U_{2}$. In fact, we have

$$
\begin{aligned}
U_{1} U_{2}^{-1} & =e^{-i e \int_{C_{1}} A(x) \cdot d x} e^{+i e \int_{C_{2}} A(x) \cdot d x} \\
& =e^{-i e \oint_{C_{1}-C_{2}} A(x) \cdot d x}=e^{-i e \iint_{S}(\nabla \times A(x)) \cdot d S} .
\end{aligned}
$$

With use of the Stokes theorem, this gives

$$
U_{1} U_{2}^{-1}=e^{-i e B x y} .
$$

To get some insight into the implication of the two path choices, we find it useful to examine the gauge-invariant photon fields corresponding to the two paths $C_{1}$ and $C_{2}$. First, for the path choice $C_{1}$, the gauge-invariant photon fields defined by Eq. 63) becomes

$$
\tilde{\boldsymbol{A}}(x, y) \equiv \tilde{\boldsymbol{A}}^{\left(C_{1}\right)}(x, y)=-B y \boldsymbol{e}_{x},
$$

which is nothing but the gauge potential in the 1st Landau gauge. On the other hand, for the choice of path $C_{2}$, the gauge-invariant photon field defined by Eq.63) reduces to

$$
\tilde{\boldsymbol{A}}(x, y) \equiv \tilde{\boldsymbol{A}}^{\left(C_{2}\right)}(x, y)=B x \boldsymbol{e}_{y},
$$

which just coincides with the gauge potential in the 2nd Landau gauge.

In the following, we adopt the 1 st form of representation (70) corresponding to the choice of path $C_{1}$. We have already pointed out that the gauge-invariant photon field corresponding to this path takes the form (76). This indicates that, in this setting of the gauge-invariant formulation, the Landau problem would reduce to that in the 1st Landau gauge. In the following, we shall show that it is indeed the case. To confirm it, we start with the original Schrödinger equation

$$
H \Psi(x, y)=E \Psi(x, y),
$$


where $H$ is the standard Landau Hamiltonian given as

$$
H=\frac{\boldsymbol{p}^{2}}{2 m_{e}}+\frac{e}{m_{e}} \boldsymbol{A} \cdot \boldsymbol{p}-i \frac{e}{2 m_{e}}(\nabla \cdot \boldsymbol{A})+\frac{e^{2}}{2 m_{e}} \boldsymbol{A}^{2} .
$$

Using the relation (70), we can transform the above eigen-equation into the equation for the gauge-invariant electron wave functions $\tilde{\Psi}^{\left(C_{1}\right)}(x, y)$ as

$$
\tilde{H}_{1} \tilde{\Psi}^{\left(C_{1}\right)}(x, y)=E \tilde{\Psi}^{\left(C_{1}\right)}(x, y)
$$

where

$$
\tilde{H}_{1} \equiv U_{1}^{-1} H U_{1}
$$

After some elementary algebra, we find that

$$
\tilde{H}_{1}=\frac{\boldsymbol{p}^{2}}{2 m_{e}}+\frac{e}{m_{e}} \tilde{\boldsymbol{A}}^{\left(C_{1}\right)} \cdot \boldsymbol{p}-i \frac{e}{2 m_{e}}\left(\nabla \cdot \tilde{\boldsymbol{A}}^{\left(C_{1}\right)}\right)+\frac{e^{2}}{2 m_{e}}\left(\tilde{\boldsymbol{A}}^{\left(C_{1}\right)}\right)^{2},
$$

where $\tilde{\boldsymbol{A}}^{\left(C_{1}\right)}$ is given by $(76)$. This means that the transformed Hamiltonian $\tilde{H}_{1}$ formally coincides with the Landau Hamiltonian in the 1st Landau gauge. Explicitly, it takes the form,

$$
\tilde{H}_{1}=\frac{1}{2 m_{e}} p_{x}^{2}+\frac{1}{2 m_{e}} p_{y}^{2}+\frac{e^{2} B^{2}}{2 m_{e}} y^{2}-\frac{e B}{m_{e}} y p_{x} .
$$

As is well-known, since this Hamiltonian does not contain $x$-dependent potential term, $\tilde{\Psi}^{\left(C_{1}\right)}(x, y)$ has the following form of solutions :

$$
\tilde{\Psi}^{\left(C_{1}\right)}(x, y)=e^{i k_{x} x} Y(y),
$$

where $k_{x}$ is the eigenvalue of the canonical momentum operator $p_{x}=-i \frac{\partial}{\partial x}$ as

$$
p_{x} \tilde{\Psi}^{\left(C_{1}\right)}(x, y)=k_{x} \tilde{\Psi}^{\left(C_{1}\right)}(x, y) .
$$

Putting (84) into (80), one finds that $Y(y)$ satisfies the following equation :

$$
\left[-\frac{1}{2 m_{e}} \frac{d^{2}}{d y^{2}}+\frac{1}{2 m_{e}}\left(k_{x}-e B y\right)^{2}\right] Y(y)=E Y(y) .
$$

This is nothing but an equation for a one-dimensional harmonic oscillator with shifted center of oscillation, whose solution is well-known. They are given by

$$
\tilde{\Psi}_{n, k_{x}}^{\left(C_{1}\right)}(x, y)=e^{i k_{x} x} Y_{n}(y),
$$

with

$$
Y_{n}(y)=\frac{1}{\sqrt{2 \pi}} N_{n} H_{n}\left(\frac{y-y_{0}}{l_{B}}\right) e^{-\frac{\left(y-y_{0}\right)^{2}}{2 l_{B}^{2}}} .
$$

Then, the original electron wave functions $\Psi(x, y)$ are given by

$$
\Psi(x, y) \equiv \Psi_{n, k_{x}}^{\left(C_{1}\right)}(x, y)=U_{1} \tilde{\Psi}_{n, k_{x}}^{\left(C_{1}\right)}(x, y) .
$$


Since the form of the original electron wave functions obtained in the above way depend on the chosen path $C_{1}$ by construction, we have explicitly written them as $\Psi_{n, k_{x}}^{\left(C_{1}\right)}(x, y)$.

Incidentally, the eigen-equation (85) for the canonical momentum can also be transformed back to an equation for the original electron wave functions. It reads as

$$
U_{1} p_{x} U_{1}^{-1} \Psi_{n, k_{x}}^{\left(C_{1}\right)}(x, y)=k_{x} \Psi_{n, k_{x}}^{\left(C_{1}\right)}(x, y) .
$$

A simple manipulation shows that

$$
U_{1} p_{x} U_{1}^{-1}=p_{x}+e A_{x}+e B y=K_{x} .
$$

Note that the r.h.s. of this equation is nothing but the $x$-component of the pseudo-momentum operator $\boldsymbol{K}$ intensively discussed by Konstantinou and Moulopoulus [8],[9] :

$$
\boldsymbol{K}=\boldsymbol{p}+e \boldsymbol{A}+e \boldsymbol{r} \times \boldsymbol{B}=\boldsymbol{\Pi}+e \boldsymbol{r} \times \boldsymbol{B} .
$$

Summarizing the analysis so far, the electron wave functions obtained in the above way are the simultaneous eigen-states of the operator $K_{x}$ and the Landau Hamiltonian $H$ :

$$
\begin{aligned}
K_{x} \Psi_{n, k_{x}}^{\left(C_{1}\right)}(x, y) & =k_{x} \Psi_{n, k_{x}}^{\left(C_{1}\right)}(x, y), \\
H \Psi_{n, k_{x}}^{\left(C_{1}\right)}(x, y) & =E_{n} \Psi_{n, k_{x}}^{\left(C_{1}\right)}(x, y)=\left(n+\frac{1}{2}\right) \omega \Psi_{n, k_{x}}^{\left(C_{1}\right)}(x, y),
\end{aligned}
$$

where $\omega=\frac{e B}{m_{e}}$. Furthermore, if we use the relation $(75)$, the eigen-functions $\Psi_{n, k_{y}}^{\left(C_{1}\right)}(x, y)$ can be written in the following form :

$$
\begin{aligned}
\Psi_{n, k_{x}}^{\left(C_{1}\right)}(x, y) & =U_{1} \tilde{\Psi}_{n, k_{x}}^{\left(C_{1}\right)}(x, y)=U_{2} e^{-i e B x y} \tilde{\Psi}_{n, k_{x}}^{\left(C_{1}\right)}(x, y) \\
& =e^{i k_{x} x} e^{-i e B x y} e^{-i e\left\{\int_{0}^{y} A_{y}\left(0, y^{\prime}\right)+\int_{0}^{x} A_{x}\left(x^{\prime}, y\right) d x^{\prime}\right\}} Y_{n}(y) .
\end{aligned}
$$

This precisely coincides with the expression given in the paper by Konstantinou and Moulopoulus by a totally different flow of the logic. As emphasized by them, a remarkable fact is that Eq. (95) combined with (88) provide us with the eigen-functions of the Landau Hamiltonian for arbitrary form of the vector potential $\boldsymbol{A}$. However, one should not forget about the fact that these solutions contain path-dependent (nonlocal) phase factor, sometimes called the Wilson line.

The case, in which the path $C_{2}$ is chosen, can be treated in a similar manner, so that we do not repeat it. After all, the electron wave functions $\Psi^{\left(C_{2}\right)}(x, y)$ obtained with this choice of path $C_{2}$ are the simultaneous eigen-states of the pseudo-momentum operator $K_{y}$ and the Landau Hamiltonian $H$ :

$$
\begin{aligned}
K_{y} \Psi_{n, k_{y}}^{\left(C_{2}\right)}(x, y) & =k_{y} \Psi_{n, k_{y}}^{\left(C_{2}\right)}(x, y), \\
H \Psi_{n, k_{y}}^{\left(C_{2}\right)}(x, y) & =E_{n} \Psi_{n, k_{y}}^{\left(C_{2}\right)}(x, y)=\left(n+\frac{1}{2}\right) \omega \Psi_{n, k_{y}}^{\left(C_{2}\right)}(x, y),
\end{aligned}
$$

where

$$
K_{y} \equiv p_{y}+e A_{y}-e B x .
$$

They are explicitly expressed as

$$
\Psi_{n, k_{y}}^{\left(C_{2}\right)}(x, y)=U_{14} e^{i k_{y} y} X_{n}(x)
$$


with

$$
U_{2}=U_{1} e^{i e B x y}=e^{i e B x y} e^{-i e\left\{\int_{0}^{x} A_{x}\left(x^{\prime}, 0\right) d x^{\prime}+\int_{0}^{y} A_{y}\left(x, y^{\prime}\right) d y^{\prime}\right\}},
$$

and

$$
X_{n}(x)=\frac{1}{\sqrt{2 \pi}} N_{n} H_{n}\left(\frac{x-x_{0}}{l_{B}}\right) e^{-\frac{\left(x-x_{0}\right)^{2}}{2 l_{B}^{2}}}
$$

Now we have two types of eigen-functions of the Landau Hamiltonian in the rectangular coordinate. The one is the simultaneous eigen-states of the pseudo momentum $K_{x}$ and the Landau Hamiltonian $H$. The other is the simultaneous eigen-states of the pseudo momentum $K_{y}$ and $H$. To understand the physical meaning of these eigen-functions as well as the meaning of gauge choice in the Landau problem, it is useful to investigate the function of three different types of momentum operators, i.e. the canonical momentum $\boldsymbol{K}^{\text {can }}$, the mechanical momentum $\boldsymbol{K}^{\text {mech }}$, and the pseudo momentum $\boldsymbol{K}$. They are given by

$$
K_{x}^{c a n} \equiv p_{x}, \quad K_{x}^{m e c h} \equiv p_{x}+e A_{x}, \quad K_{x} \equiv p_{x}+e A_{x}+e B y,
$$

and

$$
K_{y}^{c a n} \equiv p_{y}, \quad K_{y}^{m e c h} \equiv p_{y}+e A_{y}, \quad K_{y} \equiv p_{y}+e A_{y}-e B x .
$$

Summarized below is the effect of operation of the three types of operators, $K_{x}^{\text {can }}, K_{x}^{\text {mech }}$, and $K_{x}$ on the states $\left|\Psi_{n, k_{x}}^{\left(C_{1}\right)}\right\rangle$, which are simultaneous eigenstates of the operator $K_{x}$ and $H$ :

1) 1st Landau gauge : $\boldsymbol{A}_{L_{1}}=-B y \boldsymbol{e}_{x}$

$$
\begin{aligned}
K_{x}^{c a n} \Psi_{n, k_{x}}^{\left(C_{1}\right)}(x, y) & =k_{x} \Psi_{n, k_{x}}^{\left(C_{1}\right)}(x, y), \\
K_{x}^{\text {mech }} \Psi_{n, k_{x}}^{\left(C_{1}\right)}(x, y) & =\left(k_{x}-e B y\right) \Psi_{n, k_{x}}^{\left(C_{1}\right)}(x, y), \\
K_{x} \Psi_{n, k_{x}}^{\left(C_{1}\right)}(x, y) & =k_{x} \Psi_{n, k_{x}}^{\left(C_{1}\right)}(x, y) .
\end{aligned}
$$

2) 2nd Landau gauge : $\boldsymbol{A}_{L_{2}}=B x \boldsymbol{e}_{y}$

$$
\begin{aligned}
K_{x}^{c a n} \Psi_{n, k_{x}}^{\left(C_{1}\right)}(x, y) & =\left(k_{x}-e B y\right) \Psi_{n, k_{x}}^{\left(C_{1}\right)}(x, y), \\
K_{x}^{m e c h} \Psi_{n, k_{x}}^{\left(C_{1}\right)}(x, y) & =\left(k_{x}-e B y\right) \Psi_{n, k_{x}}^{\left(C_{1}\right)}(x, y), \\
K_{x} \Psi_{n, k_{x}}^{\left(C_{1}\right)}(x, y) & =k_{x} \Psi_{n, k_{x}}^{\left(C_{1}\right)}(x, y) .
\end{aligned}
$$

3) symmetric gauge : $\boldsymbol{A}_{S}=-\frac{1}{2} B y \boldsymbol{e}_{x}+\frac{1}{2} B x \boldsymbol{e}_{y}$

$$
\begin{aligned}
K_{x}^{c a n} \Psi_{n, k_{x}}^{\left(C_{1}\right)}(x, y) & =\left(k_{x}-\frac{1}{2} e B y\right) \Psi_{n, k_{x}}^{\left(C_{1}\right)}(x, y), \\
K_{x}^{m e c h} \Psi_{n, k_{x}}^{\left(C_{1}\right)}(x, y) & =\left(k_{x}-e B y\right) \Psi_{n, k_{x}}^{\left(C_{1}\right)}(x, y), \\
K_{x} \Psi_{n, k_{x}}^{\left(C_{1}\right)}(x, y) & =k_{x} \Psi_{n, k_{x}}^{\left(C_{1}\right)}(x, y) .
\end{aligned}
$$

One can see that the results of operation of the canonical momentum operator $K_{x}^{\text {can }}$ are all different depending on three gauge choices. This is thought to be a manifestation of gauge-variant nature of the canonical momentum. On the other hand, the result of operation of the operators $K_{x}^{\text {mech }}$ and $K_{x}$ are all independent of the gauge choice. It is only natural, because both of $K_{x}^{\text {mech }}$ and $K_{x}$ are manifestly gauge-invariant operators. Note, however, that the operations of $K_{x}^{\text {mech }}$ and 
Table 1: The expectation values of the three types of momentum operators, $K_{x}^{\text {can }}, K_{x}^{m e c h}$, and $K_{x}$ on the states $\left|\Psi_{n, k_{x}}^{\left(C_{1}\right)}\right\rangle$.

\begin{tabular}{cccc}
\hline & $\boldsymbol{A}_{L_{1}}=-B y \boldsymbol{e}_{x}$ & $\boldsymbol{A}_{L_{2}}=B x \boldsymbol{e}_{y}$ & $\boldsymbol{A}_{S}=-\frac{1}{2} B y \boldsymbol{e}_{x}+\frac{1}{2} B x \boldsymbol{e}_{y}$ \\
\hline$\left\langle K_{x}^{\text {can }}\right\rangle$ & $k_{x}$ & 0 & $\frac{1}{2} k_{x}$ \\
\hline$\left\langle K_{x}^{\text {mech }}\right\rangle$ & 0 & 0 & 0 \\
\hline$\left\langle K_{x}\right\rangle$ & $k_{x}$ & $k_{x}$ & $k_{x}$ \\
\hline
\end{tabular}

$K_{x}$ apparently give different answers. This last point may be seen in more obvious way through the comparison of the expectation values.

Table 1 shows the comparison of the expectation values of the three operators $K_{x}^{\text {can }}, K_{x}^{\text {mech }}$, and $K_{x}$ in the same eigenstates $\left|\Psi_{n, k_{x}}^{\left(C_{1}\right)}\right\rangle$ with different gauge choices. One confirms that the expectation values of the canonical momentum operator $K_{x}^{\text {can }}$ is gauge-dependent. On the other hand, the expectation values of the mechanical momentum operator $K_{x}^{\text {mech }}$ are independent of the gauge choices. This is again an expected result for the mechanical momentum, since it is a gauge-invariant quantity. What is less familiar is the role of another gauge-invariant momentum, which is called the pseudo momentum $K_{x}$ in the literatures [8],[9],[10]. The expectation values of this operator take the same value $k_{x}$ independently of the choices of gauge. Furthermore, it coincides with the expectation value of the canonical momentum operator $K_{x}$ in the 1st Landau gauge. This is not surprising, if we remember the following two facts. First, $\left|\Psi_{n, k_{x}}^{\left(C_{1}\right)}\right\rangle$ are the eigen-functions of the pseudo-momentum operator $K_{x}$ with the eigenvalue $k_{x}$ with any choices of gauge. Second, the pseudo-momentum operator $K_{x}$ reduces to the canonical momentum operator $K_{x}^{c a n}$ in the 1st Landau gauge. However, one should clearly recognize the fact that an expectation value $k_{x}$ of the operator $K_{x}$ is apparently different from that of the mechanical momentum operator $K_{x}^{\text {mech }}$, which is zero. As such, although $K_{x}^{\text {mech }}$ and $K_{x}$ are both gauge-invariant, they have totally different physical meaning.

To understand the importance of this difference, we first recall that an electron in a uniform magnetic field makes an circular motion around some center. Obviously, the $x$-component of the electron's momentum averaged over the period of this circular motion is zero. The fact that the expectation values of the mechanical momentum operator $K_{x}^{m e c h}$ are zero irrespectively of the gauge choices is completely consistent with this observation, which is not unrelated to the physical nature of the mechanical momentum. On the other hand, we have seen that the expectation values of the pseudo-momentum operator $K_{x}$ are $k_{x}$, which are generally nonzero. (We recall once again that the states $\left|\Psi_{n, k_{x}}^{\left(C_{1}\right)}\right\rangle$ are the eigen-states of the operator $K_{x}$ with the eigenvalue $k_{x}$. In view of the relation $k_{x}=l_{B}^{2} x_{0}$, we may as well say that $\left|\Psi_{n, k_{x}}^{\left(C_{1}\right)}\right\rangle$ are the eigen-states of the guiding-center operator $x_{0}$.) This observation indicates purely theoretical nature of the operator $K_{x}$. It would be certainly true that the pseudo momentum is a useful theoretical concept, which enables us to understand the mutual relation between different gauge choices. However, it is also true that there is no direct relation between its gauge-invariant nature and observability.

A similar analyses can be carried out for the eigenstates $\left|\Psi_{n, k_{y}}^{\left(C_{2}\right)}(x, y)\right\rangle$ and the corresponding momentum operators $K_{y}^{c a n}, K_{y}^{\text {mech }}$, and $K_{y}$. Since the general features are nothing different from the case of $\left|\Psi_{n, k_{x}}^{\left(C_{1}\right)}(x, y)\right\rangle$, except that the role of $x$ and $y$ coordinates is exchanged, we do not repeat essentially the same analyses. 


\section{2. paths in circular coordinate}

Two simple choices of path connecting the reference point $\left(r_{0}, \phi_{0}\right) \equiv(0,0)$ and the point $(r, \phi)$ in the circular coordinate representation may be given by

$$
\begin{aligned}
C_{\mathrm{I}}:(0,0) & \rightarrow(r, \phi), \\
C_{\mathrm{II}}:(0,0) & \rightarrow(r, 0) \rightarrow(r, \phi),
\end{aligned}
$$

as illustrated in Fig 2

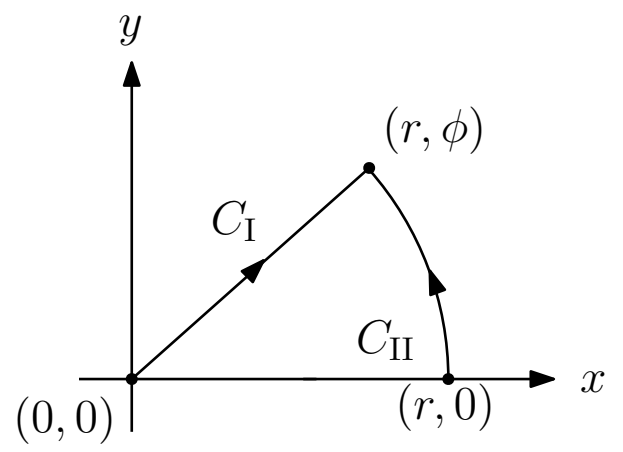

Figure 2: The two paths $C_{\mathrm{I}}$ and $C_{\mathrm{II}}$ defined in the circular coordinate system.

Selecting path $C_{\mathrm{I}}$, the relation between the original electron wave function $\Psi(r, \phi)$ and the gauge-invariant wave function $\tilde{\Psi}^{\left(C_{1}\right)}(r, \phi)$ is given by

$$
\Psi(r, \phi)=U_{I} \tilde{\Psi}^{\left(C_{I}\right)}(r, \phi),
$$

with

$$
U_{\mathrm{I}} \equiv e^{-i e \int_{C_{\mathrm{I}}} A(x) \cdot d x}=e^{-i e \int_{0}^{r} A_{r}\left(r^{\prime}, \phi\right) d r^{\prime}},
$$

where $A_{r}(r, \phi)$ is the radial component of the vector potential.

On the other hand, with the choice of the path $C_{\text {II }}$, we have

$$
\Psi(r, \phi)=U_{\mathrm{II}} \tilde{\Psi}^{\left(C_{\mathrm{II}}\right)}(r, \phi),
$$

with

$$
U_{\mathrm{II}} \equiv e^{-i e \int_{C_{\mathrm{II}}} \boldsymbol{A}(\boldsymbol{x}) \cdot d \boldsymbol{x}}=e^{-i e\left\{\int_{0}^{r} A_{r}\left(r^{\prime}, 0\right) d r^{\prime}+\int_{0}^{\phi} r A_{\phi}\left(r, \phi^{\prime}\right) d \phi^{\prime}\right\}},
$$

where $A_{\phi}(r, \phi)$ is the azimuthal component of the vector potential.

Again there is a nontrivial relation between the two operators $U_{\mathrm{I}}$ and $U_{\mathrm{II}}$, which follows from the Stokes theorem. The relation is given by

$$
U_{\mathrm{II}}^{-1} U_{\mathrm{I}}=e^{i e \oint_{C_{\mathrm{II}}-C_{\mathrm{I}}} A(x) \cdot d x}=e^{i \frac{1}{2} e B r^{2} \phi} .
$$

This identity gives a relation between the two forms of gauge-invariant electron wave functions as

$$
\tilde{\Psi}^{\left(C_{\mathrm{II}}\right)}(r, \phi)=e^{i \frac{1}{2} e B r^{2} \phi} \tilde{\Psi}^{\left(C_{\mathrm{I}}\right)}(r, \phi) .
$$


We shall come back to this relation when we discuss the gauge-invariant electron wave functions corresponding to the path $C_{\mathrm{II}}$.

To get an insight into the implication of the two path choices, it is again useful to examine the gauge-invariant photon fields corresponding to the two paths $C_{\mathrm{I}}$ and $C_{\mathrm{II}}$. First, for the path choice $C_{\mathrm{I}}$, the gauge-invariant photon fields defined by Eq. 63 becomes

$$
\tilde{\boldsymbol{A}}(r, \phi) \equiv \tilde{\boldsymbol{A}}^{\left(C_{\mathrm{I}}\right)}(r, \phi)=\frac{1}{2} B r \boldsymbol{e}_{\phi}
$$

which coincides with the gauge-potential in the symmetric gauge. On the other hand, for the path choice $C_{\mathrm{II}}$, we find that

$$
\tilde{A}(r, \phi) \equiv \tilde{A}^{\left(C_{\mathrm{II}}\right)}(r, \phi)=-B r \phi \boldsymbol{e}_{r},
$$

which is the gauge potential in the so-called Bawin-Burnel gauge [21]. (We recall that the BawinBurnel gauge is a singular and multi-valued gauge.) In any case, this simple analysis again indicates an intimate connection between the choice of gauge and the choice of path. Even stronger statement was made by Yang in a paper [22] titled "Equivalence of path dependence and gauge dependence". According to his claim, a particular path choice is nothing but a particular choice of gauge, i.e. they are totally equivalent. As we shall discuss below, our conclusion is delicately different from his conclusion. Despite the intimate connection between the path choice and the gauge choice, the choice of a particular path never prevents us from taking any other gauges.

Let us start our analysis with simpler path choice $C_{\mathrm{I}}$. With this choice of path, the original Schrödinger equation can be transformed to the following equation for $\tilde{\Psi}^{\left(C_{\mathrm{I}}\right)}$ :

$$
\tilde{H}_{\mathrm{I}} \tilde{\Psi}^{\left(C_{\mathrm{I}}\right)}(r, \phi)=E \tilde{\Psi}^{\left(C_{\mathrm{I}}\right)}(r, \phi),
$$

where

$$
\tilde{H}_{\mathrm{I}} \equiv U_{\mathrm{I}}^{-1} H U_{\mathrm{I}}
$$

After some lengthy calculation, we can show that

$$
\tilde{H}_{\mathrm{I}}=\frac{\boldsymbol{p}^{2}}{2 m_{e}}+\frac{e}{m_{e}} \tilde{\boldsymbol{A}}^{\left(C_{\mathrm{I}}\right)} \cdot \boldsymbol{p}-i \frac{e}{2 m_{e}}\left(\nabla \cdot \tilde{\boldsymbol{A}}^{\left(C_{\mathrm{I}}\right)}\right)+\frac{e^{2}}{2 m_{e}}\left(\tilde{\boldsymbol{A}}^{\left(C_{\mathrm{I}}\right)}\right)^{2},
$$

where $\tilde{\boldsymbol{A}}^{\left(C_{\mathrm{I}}\right)}$ is given by 121 . This means that the transformed Hamiltonian $\tilde{H}_{\mathrm{I}}$ formally coincides with the Landau Hamiltonian in the symmetric gauge. Explicitly, it takes the form

$$
\tilde{H}_{\mathrm{I}}=-\frac{1}{2 m_{e}} \frac{1}{r} \frac{\partial}{\partial r}\left(r \frac{\partial}{\partial r}\right)-\frac{1}{2 m_{e} r^{2}} \frac{\partial^{2}}{\partial \phi^{2}}-i \frac{e B}{2 m_{e}} \frac{\partial}{\partial \phi}+\frac{e^{2} B^{2}}{8 m_{e}} r^{2} .
$$

Since this transformed Hamiltonian contains no $\phi$-dependent potential term, the solution takes the following form :

$$
\tilde{\Psi}^{\left(C_{\mathrm{I}}\right)}(r, \phi)=e^{i m \phi} R(r),
$$

where $m$ is the eigenvalue of the canonical OAM operator $L_{z}^{c a n}$ as

$$
L_{z}^{c a n} \tilde{\Psi}^{\left(C_{\mathrm{I}}\right)}(r, \phi)=m \tilde{\Psi}^{\left(C_{\mathrm{I}}\right)}(r, \phi) .
$$

Putting (127) into (123), one finds that $R(r)$ satisfies the following equation :

$$
\left\{-\frac{1}{2 m_{e}} \frac{1}{r} \frac{d}{d r}\left(r \frac{d}{d r}\right)+\frac{1}{2 m_{e} r^{2}}\left(m+\frac{1}{2} e B r^{2}\right)^{2}\right\} R(r)=E R(r) .
$$


The solution of this equation is well known. After all, we are led to the following form of eigen-functions :

$$
\tilde{\Psi}_{n, m}^{\left(C_{1}\right)}(r, \phi)=e^{i m \phi} R_{n, m}(r)
$$

with

$$
R_{n, m}(x, y)=\frac{1}{\sqrt{2 \pi}} N_{n, m}\left(\frac{r^{2}}{2 l_{B}^{2}}\right)^{|m| / 2} e^{-\frac{r^{2}}{4 l_{B}^{2}}} L_{n-\frac{|m|+m}{2}}^{|m|}\left(\frac{r^{2}}{2 l_{B}^{2}}\right) .
$$

As a consequence, the original electron wave functions $\Psi(r, \phi)$ are given as

$$
\Psi(r, \phi) \equiv \Psi_{n, m}^{\left(C_{\mathrm{I}}\right)}(r, \phi)=U_{\mathrm{I}} \tilde{\Psi}_{n, m}^{\left(C_{\mathrm{I}}\right)}(r, \phi) .
$$

Since the form of the original electron wave functions obtained in the above way depends on the chosen path $C_{\mathrm{I}}$ by construction, we have explicitly written them as $\Psi_{n, m}^{\left(C_{\mathrm{I}}\right)}(r, \phi)$.

As before, the eigen equation (128) for the canonical OAM operator can be transformed back into the equation for the original electron wave functions. It reads as

$$
U_{\mathrm{I}} L_{z}^{c a n} U_{\mathrm{I}}^{-1} \Psi_{n, m}^{\left(C_{\mathrm{I}}\right)}(r, \phi)=m \Psi_{n, m}^{\left(C_{\mathrm{I}}\right)}(r, \phi) .
$$

A simple manipulation shows that

$$
U_{\mathrm{I}} L_{z}^{c a n} U_{\mathrm{I}}^{-1}=-i \frac{\partial}{\partial \phi}+e r A_{\phi}-\frac{1}{2} e B r^{2} .
$$

Note that the r.h.s. of this equation is nothing but the pseudo OAM operator $L_{z}$ of Konstantinou and Moulopoulus [8],[9] :

$$
L_{z}=(\boldsymbol{r} \times \boldsymbol{p})_{z}+e(\boldsymbol{r} \times \boldsymbol{A})_{z}-\frac{1}{2} e B r^{2}=(\boldsymbol{r} \times \boldsymbol{\Pi})_{z}-\frac{1}{2} e B r^{2} .
$$

To sum up, the electron wave functions obtained in the above way are the simultaneous eigenstates of the operator $L_{z}$ and the Landau Hamiltonian $H$ :

$$
\begin{aligned}
& H \Psi_{n, m}^{\left(C_{\mathrm{I}}\right)}(r, \phi)=E_{n} \Psi_{n, m}^{\left(C_{\mathrm{I}}\right)}(r, \phi)=\left(n+\frac{1}{2}\right) \omega \Psi_{n, m}^{\left(C_{\mathrm{I}}\right)}(r, \phi), \\
& L_{z} \Psi_{n, m}^{\left(C_{\mathrm{I}}\right)}(r, \phi)=m \Psi_{n, m}^{\left(C_{\mathrm{I}}\right)}(r, \phi) .
\end{aligned}
$$

If we use the relation 119 , the above eigen-functions $\Psi_{n, m}^{\left(C_{\mathrm{I}}\right)}(r, \phi)$ can also be expressed in the following form :

$$
\begin{aligned}
\Psi_{n, m}^{\left(C_{\mathrm{I}}\right)}(r, \phi) & =U_{\mathrm{I}} \tilde{\Psi}_{n, m}^{\left(C_{\mathrm{I}}\right)}(r, \phi)=U_{\mathrm{II}} e^{i \frac{1}{2} e B r^{2} \phi} \tilde{\Psi}_{n, m}^{\left(C_{\mathrm{I}}\right)}(r, \phi) \\
& =e^{i m \phi} e^{i \frac{1}{2} e B r^{2} \phi} e^{-i e\left\{\int_{0}^{r} A_{r}\left(r^{\prime}, 0\right) d r^{\prime}+\int_{0}^{\phi} r A_{\phi}\left(r, \phi^{\prime}\right) d \phi^{\prime}\right\}} R_{n, m}(r)
\end{aligned}
$$

which reproduces the expression of Konstantinou and Moulopoulus obtained with a different method. We emphasize once again that these solutions are given independently of the choice of the gauge potential $\boldsymbol{A}$.

To understand the physical implication of these eigen-states together with the meaning of the gauge choice in the Landau problem, it is instructive to investigate the function of three types of orbital angular momentum (OAM) operators, i.e. the canonical OAM, the mechanical OAM, and the pseudo OAM on these eigen-states. Shown below are the effect of operation of the three 
types of orbital angular momentum (OAM) operators, $L_{z}^{c a n}, L_{z}^{\text {mech }}$, and $L_{z}$ on the states $\left|\Psi_{n, m}^{\left(C_{\mathrm{I}}\right)}\right\rangle$, which are the simultaneous eigen-states of the operator $L_{z}$ and the Landau Hamiltonian $H$ :

1) 1st Landau gauge : $\boldsymbol{A}_{L_{1}}=-B y \boldsymbol{e}_{x}$

$$
\begin{aligned}
L_{z}^{c a n} \Psi_{n, m}^{\left(C_{\mathrm{I}}\right)}(r, \phi) & =\left(m+\frac{1}{2} e B r^{2} \cos 2 \phi\right) \Psi_{n, m}^{\left(C_{\mathrm{I}}\right)}(r, \phi), \\
L_{z}^{m e c h} \Psi_{n, m}^{\left(C_{\mathrm{I}}\right)}(r, \phi) & =\left(m+\frac{1}{2} e B r^{2}\right) \Psi_{n, m}^{\left(C_{\mathrm{I}}\right)}(r, \phi), \\
L_{z} \Psi_{n, m}^{\left(C_{\mathrm{I}}\right)}(r, \phi) & =m \Psi_{n, m}^{\left(C_{\mathrm{I}}\right)}(r, \phi) .
\end{aligned}
$$

2) 2nd Landau gauge : $\boldsymbol{A}_{L_{2}}=B x \boldsymbol{e}_{y}$

$$
\begin{aligned}
L_{z}^{c a n} \Psi_{n, m}^{\left(C_{\mathrm{I}}\right)}(r, \phi) & =\left(m-\frac{1}{2} e B r^{2} \cos 2 \phi\right) \Psi_{n, m}^{\left(C_{\mathrm{I}}\right)}(r, \phi), \\
L_{z}^{m e c h} \Psi_{n, m}^{\left(C_{\mathrm{I}}\right)}(r, \phi) & =\left(m+\frac{1}{2} e B r^{2}\right) \Psi_{n, m}^{\left(C_{\mathrm{I}}\right)}(r, \phi), \\
L_{z} \Psi_{n, m}^{\left(C_{\mathrm{I}}\right)}(r, \phi) & =m \Psi_{n, m}^{\left(C_{\mathrm{I}}\right)}(r, \phi) .
\end{aligned}
$$

3) symmetric gauge : $\boldsymbol{A}_{S}=\frac{1}{2} B r \boldsymbol{e}_{\phi}$

$$
\begin{aligned}
L_{z}^{c a n} \Psi_{n, m}^{\left(C_{\mathrm{I}}\right)}(r, \phi) & =m \Psi_{n, m}^{\left(C_{\mathrm{I}}\right)}(r, \phi), \\
L_{z}^{m e c h} \Psi_{n, m}^{\left(C_{\mathrm{I}}\right)}(r, \phi) & =\left(m+\frac{1}{2} e B r^{2}\right) \Psi_{n, m}^{\left(C_{\mathrm{I}}\right)}(r, \phi), \\
L_{z} \Psi_{n, m}^{\left(C_{\mathrm{I}}\right)}(r, \phi) & =m \Psi_{n, m}^{\left(C_{\mathrm{I}}\right)}(r, \phi) .
\end{aligned}
$$

First, one notices that the result of operation of the canonical OAM operator $L_{z}^{\text {can }}$ are all different, depending on the three gauge choices. This is again a manifestation of the gaugedependent nature of the canonical OAM. In contrast, reflecting the fact that the mechanical OAM operator $L_{z}^{\text {mech }}$ and the pseudo OAM operator $L_{z}$ are both gauge-invariant, the result of operation of these operators are all the same irrespectively of the gauge choices. Note, however, that the operations of $L_{z}^{\text {mech }}$ and $L_{z}$ give different answers.

Table 2: The expectation values of the three types of OAM operators, $L_{z}^{c a n}, L_{z}^{\text {mech }}$, and $L_{z}$ on the states $\left|\Psi_{n, m}^{\left(C_{\mathrm{I}}\right)}\right\rangle$.

\begin{tabular}{cccc}
\hline & $\boldsymbol{A}_{L_{1}}=-B y \boldsymbol{e}_{x}$ & $\boldsymbol{A}_{L_{2}}=B x \boldsymbol{e}_{x}$ & $\boldsymbol{A}_{S}=\frac{1}{2} B r \boldsymbol{e}_{\phi}$ \\
\hline$\left\langle L_{z}^{\text {can }}\right\rangle$ & $m$ & $m$ & $m$ \\
\hline$\left\langle L_{z}^{\text {mech }}\right\rangle$ & $2 n+1$ & $2 n+1$ & $2 n+1$ \\
\hline$\left\langle L_{z}\right\rangle$ & $m$ & $m$ & $m$ \\
\hline
\end{tabular}

To see it more transparently, let us compare in Table 2 the expectation values of the three OAM operators $L_{z}^{c a n}, L_{z}^{\text {mech }}$, and $L_{z}$ between the eigen-states $\left|\Psi_{n, m}^{\left(C_{\mathrm{I}}\right)}\right\rangle$ with different gauge choices. One observes that the expectation values of any of the three OAM operators $L_{z}^{\text {can }}, L_{z}^{\text {mech }}$, and $L_{z}$ 
do not depend on the choices of gauge. In particular, one sees that the expectation values of the canonical OAM operator and the pseudo OAM operator are both $m$. This coincidence is natural in the symmetric gauge. It is because the pseudo OAM operator reduces to the canonical OAM operator with this gauge choice. A little nontrivial is the observation that the expectation value of the canonical OAM operator also coincides with $m$ in other two gauges than the symmetric gauge. This should be contrasted with the fact that the expectation value of the canonical momentum operators $K_{x}^{c a n}=p_{x}$ depends on the gauge choice. This difference is related to the fact that there are two canonical momentum operators $p_{x}$ and $p_{y}$, which cannot be diagonalized simultaneously, while there is only one canonical OAM operator $L_{z}^{c a n}$. As a matter of course, this fact alone cannot explain why the expectation value of $L_{z}^{c a n}$ does not depend on the gauge choice. In the case of the two Landau gauges, which we have investigated in the present paper, the reason can be relatively easily understood. It is because the matrix element of the terms containing $\cos 2 \phi$ in (139) and (142) between the eigen-functions $\left|\Psi_{n, m}^{\left(C_{I}\right)}\right\rangle$ vanish after integration over the azimuthal angle $\phi$. A natural question is what happens with other gauges than the two Landau gauges. We found that the expectation value of the canonical OAM operator between the eigenstates $\left|\Psi_{n, m}^{\left(C_{I}\right)}\right\rangle$ is given by $m$ in arbitrary single-valued (or regular) gauges. An essential ingredient leading to this result is the periodicity of the wave functions $\Psi_{n, m}^{\left(C_{I}\right)}(r, \phi)$ as well as the gauge field configuration $\boldsymbol{A}(r, \phi)$ with respect to the angle $\phi$ with the $2 \pi$ period. To our best knowledge, this fact has not been shown explicitly before. We therefore think it useful to give its proof in Appendix D.

Turning to the expectation value of the mechanical OAM operator, we see that it is given by $2 n+1$, with $n$ being the familiar Landau quantum number, irrespectively of the gauge choice. Since this quantum number appears in the expression of the Landau energy levels $E_{n}=\left(n+\frac{1}{2}\right) \omega$, it clearly corresponds to an observable. This is not the case for the quantum number $m$. As is well-known, each Landau level with a fixed quantum number $n$ has infinitely many degeneracies with different values of $m$. This means that, at least in the Landau problem, the quantum number $m$ and therefore the canonical OAM as well as the pseudo OAM are not such quantities, which have direct connection with observables.

Also very important to recognize is the fact that the simultaneous eigen-states of $L_{z}$ and $H$, which we have discussed above, do not reduce to the standardly-known eigen-states in the Landau gauge even in the limit of Landau gauge fixing in the above gauge-invariant formulation. To confirm it, we recall that, in the gauge-invariant formulation, the simultaneous eigen-states of $L_{z}$ and $H$ are given as

$$
\Psi_{n, m}^{\left(C_{\mathrm{I}}\right)}(r, \phi)=U_{\mathrm{I}} e^{i m \phi} R_{n, m}(r)=e^{i m \phi} e^{-i e \int_{0}^{r} A_{r}\left(r^{\prime}, \phi\right) d r^{\prime}} R_{n, m}(r),
$$

where $R_{m, n}(r)$ is given by 131 . With the choice of the symmetric gauge, which amounts to taking $A_{r}=0, A_{\phi}=\frac{1}{2} B r$, the above eigen-functions reduce to

$$
\Psi_{n, m}^{\left(C_{\mathrm{I}}\right)}(r, \phi) \rightarrow e^{i m \phi} R_{n, m}(r)
$$

which is nothing but the standard eigen-functions of the Landau Hamiltonian in the symmetric gauge. On the other hand, in the limit of taking the 2nd type of Landau gauge, which amounts to taking $A_{r}=r B \cos \phi \sin \phi, A_{\phi}=r B \cos ^{2} \phi$, the eigen-functions reduces to

$$
\Psi_{n, m}^{\left(C_{\mathrm{I}}\right)}(r, \phi) \rightarrow e^{i m \phi} e^{-i \frac{1}{2} e B x y} R_{n, m}(r)
$$

Clearly, they do not coincides with the standard eigen-functions of the Landau Hamiltonian in the Landau gauge. The truth is that, as we have already discussed in sect 1, the r.h.s. of the 
above equation is proportional to a particular superposition of the standard eigen-functions in the Landau gauge. Namely, the eigen-function $\Psi_{n, m}^{\left(C_{1}\right)}(r, \phi)$ in the gauge-invariant formulation reduces in the Landau gauge limit to the following functions :

$$
\Psi_{n, m}^{\left(C_{\mathrm{I}}\right)}(r, \phi) \rightarrow e^{-i \frac{1}{2} e B x y} \int d k_{y} U_{n, m}\left(x_{0}\right) \Psi_{n, k_{y}}(x, y),
$$

where $\Psi_{n, k_{y}}(x, y)$ is the standard eigen-functions of the Landau Hamiltonian in the 1st Landau gauge, while the weight function $U_{n, m}\left(x_{0}\right)$ of the superposition is given as the matrix element of the gauge transformation operator between the standard eigen-states in the Landau gauge and the standard eigen-functions in the symmetric gauge as shown in sect 1 .

\subsection{A short summary on the issue of gauge choice in the Landau problem}

The delicacy of the gauge choice in the Landau problem should be clear by now. In the standard treatment, the choice of gauge and that of coordinate system is inseparably connected. This is so because, if we choose the Landau gauge, the rectangular-coordinate description is more natural and economical, since the gauge potential in this Hamiltonian depends on either of coordinate $x$ or $y$ only. On the other hand, if the symmetric gauge is chosen, the gauge potential has an axial symmetry and it has azimuthal component only. Therefore, the circular coordinate choice is more convenient to work with.

For getting still deeper insight into the delicacy of the gauge choice in the Landau problem, more powerful is the gauge-invariant formulation of the same problem. For the Landau problem, two different types of gauge-invariant formulation are possible. The first is the gauge-invariant formulation of Konstantinou and Moulopoulos [8], [9] or of Haugset et al. [17]. In this formulation, what plays an important role is the existence of the two pseudo-momentum operator $\boldsymbol{K}$ and the pseudo OAM operator $L_{z}$, which are both gauge-invariant objects. A remarkable fact is that we can obtain the simultaneous eigen-states of one of these operators and the Landau Hamiltonian without fixing gauge. This is assured by the fact that the gauge-invariant operator $\boldsymbol{K}$ as well as the gauge-invariant operator $L_{z}$ commute with the Landau Hamiltonian [8], [9],

$$
[\boldsymbol{K}, H]=0, \quad\left[L_{z}, H\right]=0 .
$$

These operators are not mutually commutable, however,

$$
\left[K_{x}, K_{y}\right] \neq 0, \quad\left[K_{x}, L_{z}\right] \neq 0, \quad\left[K_{y}, L_{z}\right] \neq 0 .
$$

The possibilities are therefore to look for simultaneous eigen-states of $K_{x}$ and $H$, or $K_{y}$ and $H$, or $L_{z}$ and $H$. The first two choices give eigen-functions, which can be expressed in terms of Hermite polynomials, while the third choice gives eigen-functions, which can be expressed with associated Laguerre polynomials. As emphasized by Haugset et al. [17], "Which of eigenfunctions one wants to work with is basically not equivalent to the choice of gauge, but rather which operator one wants to have diagonalized." An advantage of working in the third type of eigen-function is that the axial symmetry of the problem is maintained and the meaning of the angular momentum operators becomes clear. In fact, as we have seen, to give a clear meaning to these quantities, we had to consider a superposition of the eigen-states if we stick to working in the first two types of formulations.

Actually, we have solved the Landau problem based on another gauge-invariant formulation, i.e. DeWitt's formalism. This formulation has broader utility than the above-mentioned one, 
since the existence of the local and gauge-invariant pseudo momenta and pseudo OAM is not assumed as a prerequisite of formulation. (As we shall see in later section, this feature is important for our discussion of the nucleon spin decomposition problem, because no analogous local quantities like the ones $\boldsymbol{K}$ and $L_{z}$ are not known to exist in this case.) Instead, what is essential in DeWitt's formalism is the freedom in the choice of path. As we have shown, with the choice of the path $C_{1}$ in the rectangular coordinate, for example, the gauge-invariant photon field reduces to the vector potential in the 1 st Landau gauge. In this sense, the choice of the path $C_{1}$ is intimately connected with the choice of the 1 st Landau gauge. Nonetheless, this choice of path is not totally equivalent to taking the 1st Landau gauge. Within the gauge-invariant formulation of DeWitt, we still have a freedom to take other gauges like the 2nd Landau gauge and the symmetric gauge. A natural question is therefore what is special with the choice of the path $C_{1}$. The answer is already clear. It is the realization of translational symmetry with respect to the $x$-axis. In fact, as one can see clearly from the fourth column of Table 1, this symmetry manifests itself in the gauge-choice independence of the expectation value of the pseudo-momentum operator $K_{x}$ or the g.i.c. momentum operator in our terminology.

Similarly, if we take the path $C_{I}$ in the cylindrical coordinate, it amounts to respecting the axial symmetry or the rotational symmetry around the $z$-axis. Again, it is not equivalent to taking the symmetric gauge, since we can still choose other gauges like the two Landau gauges. In this case, the axial symmetry manifests in the gauge-choice independence of the expectation value of the pseudo OAM operator or the g.i.c. OAM in our terminology, as can be seen from the fourth column of Table 2. In this way, we arrive at an important conclusion. Choosing a particular path is equivalent to deciding what symmetry is to be respected in the physics of our interest. We emphasize that we have proven this fact based on a concrete example, which makes full use of the analytically-obtained wave functions of the Landau problem within DeWitt's formalism.

\subsection{The choice of path $C_{I I}$ and the multi-valued gauge}

Before ending this section, for the sake of completeness, we briefly discuss what happens if we choose another path $C_{\mathrm{II}}$ in the cylindrical coordinate for defining the gauge-invariant electron wave functions. As already pointed out, the gauge-invariant wave functions $\tilde{\Psi}^{\left(C_{\mathrm{II}}\right)}(r, \phi)$ corresponding to the path $C_{\mathrm{II}}$ are related to the wave functions $\tilde{\Psi}^{\left(C_{\mathrm{I}}\right)}(r, \phi)$ by Eq. (120), i.e. as $\tilde{\Psi}^{\left(C_{\mathrm{II}}\right)}(r, \phi)=e^{\frac{1}{2} i e B r^{2} \phi} \tilde{\Psi}^{\left(C_{\mathrm{I}}\right)}(r, \phi)$. Since $\tilde{\Psi}^{\left(C_{\mathrm{I}}\right)}(r, \phi)$ is a periodic function of $\phi$ with a period $2 \pi$, i.e.

$$
\tilde{\Psi}^{\left(C_{\mathrm{I}}\right)}(r, \phi+2 \pi)=\tilde{\Psi}^{\left(C_{\mathrm{I}}\right)}(r, \phi),
$$

it follows that the wave functions $\tilde{\Psi}^{\left(C_{\mathrm{II}}\right)}(r, \phi)$ satisfy the following unusual boundary condition,

$$
\tilde{\Psi}^{\left(C_{\mathrm{II}}\right)}(r, \phi+2 \pi)=e^{i \pi e B r^{2}} \tilde{\Psi}^{\left(C_{\mathrm{II}}\right)}(r, \phi),
$$

which means that they are multi-valued functions with respect to the variable $\phi$. We point out that this is consistent with the fact that the gauge-invariant photon field corresponding to the path $C_{\text {II }}$ is given by (122), which is just the vector potential in the (multi-valued) Bawin-Burnel (BB) gauge.

In any case, using the relation (120), the electron wave-functions corresponding to the path choice $C_{\mathrm{II}}$ can be readily obtained as

$$
\begin{aligned}
& \Psi_{n, m}^{\left(C_{\mathrm{II}}\right)}(r, \phi)=U_{\mathrm{II}} \tilde{\Psi}_{n, m}^{\left(C_{\mathrm{II}}\right)}(r \phi)=U_{\mathrm{II}} e^{i \frac{1}{2} e B r^{2} \phi} \tilde{\Psi}_{n, m}^{\left(C_{\mathrm{I}}\right)}(r \phi) \\
&=U_{\mathrm{II}} e^{i \frac{1}{2} e B r^{2} \phi} e^{i m \phi} R_{n, m}(r) \\
&=e^{i\left(m+\frac{1}{2} e B r^{2}\right) \phi} e^{-i e\left\{\int_{0}^{r} A_{r}\left(r^{\prime}, 0\right) d r^{\prime}+\int_{0}^{\phi} r A_{\phi}\left(r, \phi^{\prime}\right) d \phi^{\prime}\right\}} R_{n, m}(r), \\
& 23
\end{aligned}
$$


where $R_{n, m}(r)$ is given by (131). Again, one may be interested in the result of operation of the three types of orbital angular momentum operators, i.e. $L_{z}^{\text {can }}, L_{z}^{\text {mech }}$, and $L_{z}$ on the states $\left|\Psi_{n, m}^{\left(C_{\mathrm{II}}\right)}\right\rangle$. Using the circular coordinate representation of these OAMs given by

$$
\begin{aligned}
L_{z}^{c a n} & =-i \frac{\partial}{\partial \phi}, \\
L_{z}^{\text {mech }} & =-i \frac{\partial}{\partial \phi}+e r A_{\phi}, \\
L_{z} & =-i \frac{\partial}{\partial \phi}+e r A_{\phi}-\frac{1}{2} e B r^{2},
\end{aligned}
$$

one is led to the following answer :

1) 1st Landau gauge : $\boldsymbol{A}_{L_{1}}=-B y \boldsymbol{e}_{x}$

$$
\begin{aligned}
L_{z}^{c a n} \Psi_{n, m}^{\left(C_{\mathrm{II}}\right)}(r, \phi) & =\left(m+\frac{1}{2} e B r^{2} \cos 2 \phi\right) \Psi_{n, m}^{\left(C_{\mathrm{II}}\right)}(r, \phi), \\
L_{z}^{m e c h} \Psi_{n, m}^{\left(C_{\mathrm{II}}\right)}(r, \phi) & =\left(m+\frac{1}{2} e B r^{2}\right) \Psi_{n, m}^{\left(C_{\mathrm{II}}\right)}(r, \phi), \\
L_{z} \Psi_{n, m}^{\left(C_{\mathrm{II}}\right)}(r, \phi) & =m \Psi_{n, m}^{\left(C_{\mathrm{II}}\right)}(r, \phi) .
\end{aligned}
$$

2) 2nd Landau gauge : $\boldsymbol{A}_{L_{2}}=B x \boldsymbol{e}_{y}$

$$
\begin{aligned}
L_{z}^{c a n} \Psi_{n, m}^{\left(C_{\mathrm{II}}\right)}(r, \phi) & =\left(m-\frac{1}{2} e B r^{2} \cos 2 \phi\right) \Psi_{n, m}^{\left(C_{\mathrm{II}}\right)}(r, \phi), \\
L_{z}^{m e c h} \Psi_{n, m}^{\left(C_{\mathrm{II}}\right)}(r, \phi) & =\left(m+\frac{1}{2} e B r^{2}\right) \Psi_{n, m}^{\left(C_{\mathrm{II}}\right)}(r, \phi), \\
L_{z} \Psi_{n, m}^{\left(C_{\mathrm{II}}\right)}(r, \phi) & =m \Psi_{n, m}^{\left(C_{\mathrm{II}}\right)}(r, \phi) .
\end{aligned}
$$

3) symmetric gauge : $\boldsymbol{A}_{S}=\frac{1}{2} B r \boldsymbol{e}_{\phi}$

$$
\begin{aligned}
L_{z}^{c a n} \Psi_{n, m}^{\left(C_{\mathrm{II}}\right)}(r, \phi) & =m \Psi_{n, m}^{\left(C_{\mathrm{II}}\right)}(r, \phi), \\
L_{z}^{m e c h} \Psi_{n, m}^{\left(C_{\mathrm{II}}\right)}(r, \phi) & =\left(m+\frac{1}{2} e B r^{2}\right) \Psi_{n, m}^{\left(C_{\mathrm{II}}\right)}(r, \phi), \\
L_{z} \Psi_{n, m}^{\left(C_{\mathrm{II}}\right)}(r, \phi) & =m \Psi_{n, m}^{\left(C_{\mathrm{II}}\right)}(r, \phi) .
\end{aligned}
$$

4) Bawin-Burnel (BB) gauge : $\boldsymbol{A}_{B B}=-B r \boldsymbol{e}_{r}$

$$
\begin{aligned}
L_{z}^{c a n} \Psi_{n, m}^{\left(C_{\mathrm{II}}\right)}(r, \phi) & =\left(m+\frac{1}{2} e B r^{2}\right) \Psi_{n, m}^{\left(C_{\mathrm{II}}\right)}(r, \phi), \\
L_{z}^{m e c h} \Psi_{n, m}^{\left(C_{\mathrm{II}}\right)}(r, \phi) & =\left(m+\frac{1}{2} e B r^{2}\right) \Psi_{n, m}^{\left(C_{\mathrm{II}}\right)}(r, \phi), \\
L_{z} \Psi_{n, m}^{\left(C_{\mathrm{II}}\right)}(r, \phi) & =m \Psi_{n, m}^{\left(C_{\mathrm{II}}\right)}(r, \phi) .
\end{aligned}
$$

One confirms that the results of operation of $L_{z}^{\text {mech }}$ and $L_{z}$ do not depend on the gauge-field configuration, i.e. they are the same for all of the choices $\boldsymbol{A}_{L_{1}}, \boldsymbol{A}_{L_{2}}, \boldsymbol{A}_{S}$, and $\boldsymbol{A}_{B B}$. However, this is not the case for the canonical OAM operator $L_{z}^{\text {can }}$, which is a gauge-variant operator. 
Table 3: The expectation values of the three types of OAM operators, $L_{z}^{\text {can }}, L_{z}^{m e c h}$, and $L_{z}$ on the states $\left|\Psi_{n, m}^{\left(C_{\mathrm{II}}\right)}\right\rangle$.

\begin{tabular}{ccccc}
\hline & $\boldsymbol{A}_{L_{1}}=-B y \boldsymbol{e}_{x}$ & $\boldsymbol{A}_{L_{2}}=B x \boldsymbol{e}_{x}$ & $\boldsymbol{A}_{S}=\frac{1}{2} B r \boldsymbol{e}_{\phi}$ & $\boldsymbol{A}_{B B}=-B r \phi \boldsymbol{e}_{r}$ \\
\hline$\left\langle L_{z}^{\text {can }}\right\rangle$ & $m$ & $m$ & $m$ & $2 n+1$ \\
\hline$\left\langle L_{z}^{\text {mech }}\right\rangle$ & $2 n+1$ & $2 n+1$ & $2 n+1$ & $2 n+1$ \\
\hline$\left\langle L_{z}\right\rangle$ & $m$ & $m$ & $m$ & $m$ \\
\hline
\end{tabular}

Also interesting to see is the expectation values of the three OAM operators between the eigenstates $\left|\Psi_{n, m}^{\left(C_{\mathrm{II}}\right)}\right\rangle$. They are shown in Table 3. One finds that the expectation values of $L_{z}^{\text {mech }}$ are all $2 n+1$ irrespectively of the choices of the gauge potential. On the other hand, the expectation values of $L_{z}$ are all $m$, independently of the choices of gauge potential. However, the expectation values of $L_{z}^{c a n}$ is $2 n+1$ in the BB gauge, whereas they are $m$ in other three gauges. Thus, for the canonical OAM operator, we find that even the expectation values are generally dependent on the gauge choice. Also very interesting is the fact that the expectation values of $L_{z}^{c a n}$ and that of $L_{z}$ do not coincide in the BB gauge. The gauge-choice independence of the pseudo-OAM operator $L_{z}$ can be interpreted as showing its unique theoretical nature as the generator of rotation around the $z$-axis [8],[9].

\section{On the concept of gauge-invariant canonical orbital angular momentum}

We have reconfirmed that the pseudo momentum $\boldsymbol{K}$ and the pseudo OAM $L_{z}$ defined by

$$
\begin{aligned}
\boldsymbol{K} & \equiv \boldsymbol{\Pi}+e \boldsymbol{r} \times \boldsymbol{B}, \\
L_{z} & \equiv(\boldsymbol{r} \times \boldsymbol{\Pi})_{z}-\frac{1}{2} e B r^{2},
\end{aligned}
$$

play important roles in the gauge-invariant formulation of the Landau problem [8],[9]. A remarkable features of these quantities are that they are apparently gauge-invariant and besides they reduce to the canonical momentum and canonical OAM in suitable gauges. This reminds us of the concept of gauge-invariant canonical (g.i.c.) momentum and the gauge-invariant canonical (g.i.c.) OAM, which were advocated by Chen et al., and intensively discussed in the context of the nucleon spin decomposition problem as well as the photon spin decomposition problem [11],[12]. Their basic idea starts with decomposing the gauge field into what-they-call the "physical" component and the "pure-gauge" component as

$$
\boldsymbol{A}=\boldsymbol{A}_{\text {phys }}+\boldsymbol{A}_{\text {pure }} .
$$

If one restricts to the case of quantum electrodynamics, which is an abelian gauge theory, their proposal reduces to the decomposition of the total photon field (or the vector potential) into the following two components,

$$
\boldsymbol{A}=\boldsymbol{A}_{\perp}+\boldsymbol{A}_{\|}
$$

where $\boldsymbol{A}_{\perp}$ and $\boldsymbol{A}_{\|}$are respectively the familiar transverse and longitudinal components of the photon field, satisfying the conditions $\nabla \cdot \boldsymbol{A}_{\perp}=0$ and $\nabla \times \boldsymbol{A}_{\|}=0$. Once the Lorentz frame is 
fixed, this transverse-longitudinal decomposition is known to be unique owing to the Helmholtz theorem, provided that the vector potential $\boldsymbol{A}$ damps fast enough at the spatial infinity. Also noteworthy is the gauge transformation property of the two components $\boldsymbol{A}_{\perp}$ and $\boldsymbol{A}_{\|}$. Under general gauge transformations $\boldsymbol{A} \rightarrow \boldsymbol{A}+\nabla \chi$, it can be shown that they transform as $\boldsymbol{A}_{\perp} \rightarrow \boldsymbol{A}_{\perp}$ and $\boldsymbol{A}_{\|} \rightarrow \boldsymbol{A}_{\|}+\nabla \chi$. That is, the transverse component is gauge-invariant, while the longitudinal component carries the residual gauge degrees of freedom. From this fact, it immediately follows that the operators defined by

$$
\begin{aligned}
& \boldsymbol{p}^{\text {g.i.c. }} \equiv \boldsymbol{p}+e \boldsymbol{A}_{\|}, \\
& L_{z}^{\text {g.i.c. }} \equiv\left[\boldsymbol{r} \times\left(\boldsymbol{p}+e \boldsymbol{A}_{\|}\right)\right]_{z},
\end{aligned}
$$

transform just in the same way as

$$
\begin{aligned}
& \boldsymbol{p}^{\text {mech }}=\boldsymbol{p}+e \boldsymbol{A}, \\
& L_{z}^{\text {mech }}=[\boldsymbol{r} \times(\boldsymbol{p}+e \boldsymbol{A})]_{z},
\end{aligned}
$$

which in turn means that $\boldsymbol{p}^{\text {g.i.c. }}$ as well as $L_{z}^{\text {g.i.c. }}$ are both gauge-invariant. Besides, if the gauge degrees of freedom are eliminated, which allows to set $\boldsymbol{A}_{\|}=0$, they clearly reduce to the ordinary canonical momentum and canonical OAM operators. For this reason, these quantities are often called the gauge-invariant canonical (g.i.c.) momentum and the gauge-invariant canonical (g.i.c.) OAM in the literatures [1], [3].

As can be easily verified, the gauge potential $\boldsymbol{A}_{S}$ in the symmetric gauge satisfies the transverse condition $\nabla \cdot \boldsymbol{A}_{S}=0$. (Since this condition is just the same as the Coulomb gauge condition, the symmetric gauge is sometimes called the Coulomb gauge in some paper [23].) Then, one might be tempted to identity the gauge potential in the symmetric gauge with the transverse component of the vector potential. With this identification, we have

$$
\left(\boldsymbol{r} \times \boldsymbol{A}_{\perp}\right)_{z}=\left(\boldsymbol{r} \times \boldsymbol{A}_{S}\right)_{z}=\frac{1}{2} B r^{2} .
$$

As a consequence, the pseudo OAM operator $L_{z}$ reduces to

$$
\begin{aligned}
L_{z} & =(\boldsymbol{r} \times \boldsymbol{p})_{z}+e\left(\boldsymbol{r} \times \boldsymbol{A}_{\perp}\right)_{z}+e\left(\boldsymbol{r} \times \boldsymbol{A}_{\|}\right)_{z}-\frac{1}{2} e B r^{2} \\
& =\left[\boldsymbol{r} \times\left(\boldsymbol{p}+e \boldsymbol{A}_{\|}\right)\right]_{z}=\left(\boldsymbol{r} \times \boldsymbol{D}_{\text {pure }}\right)_{z}=L_{z}^{\text {g.i.c }}
\end{aligned}
$$

with $\boldsymbol{D}_{\text {pure }} \equiv \boldsymbol{p}+\boldsymbol{e} \boldsymbol{A}_{\|}$being the so-called pure-gauge covariant derivative. This last quantity is nothing but the gauge-invariant canonical OAM of Chen et al.

Unfortunately, the condition of the Helmholtz theorem is not satisfied in the Landau problem, since the magnetic field spreads over the whole plane. As a consequence, the transverselongitudinal decomposition cannot be carried out uniquely and the above identification is also not unique. In fact, one can easily verify that the vector potentials $\boldsymbol{A}_{L_{1}}, \boldsymbol{A}_{L_{2}}$, and $\boldsymbol{A}_{S}$ in the 1st and 2nd Landau gauges and the symmetric gauge, all satisfy the transverse condition, i.e. $\nabla \cdot \boldsymbol{A}_{L_{1}}=\nabla \cdot \boldsymbol{A}_{L_{2}}=\nabla \cdot \boldsymbol{A}_{S}=0$. Still, as we have discussed in sect 1 , if one wants to analyze the orbital angular momentum of the electron, the symmetric gauge appears to be the most natural and convenient choice. (As a matter of course, we expect that the genuine physical observables like the Landau levels are independent of the choice of gauges.) This is obviously related to the fact that, even though the uniform magnetic field in the Landau problem does not have any 
preferred symmetry axis, the motion of the electron in this magnetic field is a circular motion around some center, which means that there is an apparent cylindrical symmetry in physics.

What if there is an axial symmetry in the magnetic field distribution from the outset, then ? In such a situation, the choice of symmetric gauge seems almost mandatory, or at least most convenient. As an example, let us consider an infinitely long solenoid along the $z$-direction with $R$ being the radius of the cross section of it. In this setting, there is a uniform magnetic field only inside the solenoid, while there is no magnetic field outside it, such that

$$
\boldsymbol{B}(\boldsymbol{x})=B_{0} \theta(R-r) \boldsymbol{e}_{z} .
$$

Li et al. argued that the transverse component $\boldsymbol{A}_{\perp}$ of the vector potential for this magnetic field can uniquely be determined by the Helmholtz theorem [24]. According to the Helmholtz theorem, $\boldsymbol{A}_{\perp}$ can formally be expressed as

$$
\begin{aligned}
\boldsymbol{A}_{\perp}(\boldsymbol{x}) & =\nabla \times \frac{1}{4 \pi} \int d^{3} x^{\prime} \frac{\nabla^{\prime} \times \boldsymbol{A}\left(\boldsymbol{x}^{\prime}\right)}{\left|\boldsymbol{x}-\boldsymbol{x}^{\prime}\right|} \\
& =\nabla \times \frac{1}{4 \pi} \int d^{3} x^{\prime} \frac{\boldsymbol{B}\left(\boldsymbol{x}^{\prime}\right)}{\left|\boldsymbol{x}-\boldsymbol{x}^{\prime}\right|}=\nabla \times \frac{B_{0}}{4 \pi} \boldsymbol{e}_{z} \int_{\left|x^{\prime}\right| \leq R} \frac{d^{3} x^{\prime}}{\left|\boldsymbol{x}-\boldsymbol{x}^{\prime}\right|}
\end{aligned}
$$

Actually, for an infinitely long solenoid, the above integral is logarithmically divergent. To regulate the integral, Li et al. first consider a solenoid with large but finite length $L[24]$. For this solenoid, they showed that

$$
\int_{\left|x^{\prime}\right|<R} \frac{d^{3} x^{\prime}}{\left|\boldsymbol{x}-\boldsymbol{x}^{\prime}\right|}=\pi R^{2} \ln 4 L^{2}+\pi R^{2} \ln R^{2}-\pi R^{2}+\pi r^{2}+O\left(\frac{1}{L^{2}}\right)
$$

for $r<R$, and

$$
\int_{\left|x^{\prime}\right|<R} \frac{d^{3} x^{\prime}}{\left|\boldsymbol{x}-\boldsymbol{x}^{\prime}\right|}=\pi R^{2} \ln 4 L^{2}+\pi R^{2} \ln r^{2}+O\left(\frac{1}{L^{2}}\right),
$$

for $r>R$. Inserting these expressions into [183), and then taking the $L \rightarrow \infty$ limit, they obtain

$$
\boldsymbol{A}_{\perp}(\boldsymbol{x})=\left\{\begin{array}{cc}
\frac{1}{2} B r \boldsymbol{e}_{\phi} & (r \leq R), \\
\frac{1}{2} \frac{B R^{2}}{r} \boldsymbol{e}_{\phi} & (r>R)
\end{array}\right.
$$

which confirms that the Helmholtz theorem uniquely fix the transverse component of the vector potential. Very interestingly, if one further takes the limit $R \rightarrow \infty$, which amounts to considering a uniform magnetic field spread over the whole $x$ - $y$ plane, one obtains

$$
\boldsymbol{A}_{\perp}(\boldsymbol{x}) \rightarrow \frac{1}{2} B r \boldsymbol{e}_{\phi}
$$

This is nothing but the gauge potential of the symmetric gauge in the Landau problem.

Our analysis shows that the canonical OAM is an important quantity that characterizes the quantum mechanical eigen-states of a Hamiltonian with cylindrical symmetry. Nevertheless, it does not correspond to any observable at least in the Landau problem. However, we refrain from concluding that the canonical OAM can never be observed. One of the reasons is that we are aware of the recent research progress, which suggests possible observability of the canonical OAM [25],[26]. The point is to consider the motion of electron along the direction of the uniform magnetic field, which was neglected in most analyses of the Landau problem in the past. 
From the technical viewpoint, the idea is motivated by the development of the new experimental technique, i.e. the generation of the so-called electron vortex beam by utilizing the transmission electron microscopes (TEM). They proposed to use this electron vortex beam running along the direction of the magnetic field, which is supposed to be practically uniform as compared with the size of the electron vortex beam. According to them, this setting enables real-space elucidation of individual Landau level, which is characterized by two quantum numbers, i.e. the Landau quantum number $n$ and the eigen-value $m$ of the canonical OAM operator. In fact, they showed that, while propagating along the direction of the magnetic field, the Landau electrons receive characteristic rotation with three different angular velocities, depending on the eigen-value $m$ of the canonical OAM. This rotation during the propagation along the $z$-direction can be measured experimentally by using statistical averaging over many identical single-particle events. This then enables to get direct relation between the canonical OAM and an observable. It is a remarkable findings, in the sense that it sheds light on the so-far hidden $m$-dependent rotational dynamics of the quantum Landau states for the first time. However, it also appears to bring about a new question on the interpretation of the widely-accepted fundamental principle of physics, i.e. the gauge principle. In fact, according to the gauge principle, observables must be gauge-invariant, but the canonical OAM is not a gauge-invariant quantity.

\section{Remarks on the issue of gauge choice in the nucleon spin decomposition problem}

In the discussion of the gauge-invariant formulation of the Landau problem, we have pointed out that there are in principle infinitely many choices of path for defining the gauge-invariant electron and photon fields and besides that there is an intimate connection between the choice of path and the choice of gauge. Once, Belinfante showed that, by averaging over path-dependent potential over the direction of all straight line paths at constant time converging to the point where the gauge potential is to be calculated, one is led to the gauge potential in the Coulomb gauge [27]. On the other hand, Rohrlich and Strocchi applied a similar averaging procedure over a covariant path, thereby obtaining the gauge potential in the Lorentz gauge [28]. Since the Coulomb gauge is one of the most important gauges, which falls into the class of the socalled physical gauges, we briefly introduce Belinfante's argument [27]. (The physical gauge is a particular class of gauges, which maintains only two physical degrees of freedom of the massless vector field. The greatest advantage of the physical gauge is that they do not need to introduce the Hilbert space with indefinite metric, which is unavoidable in the quantization with the covariant gauge choice like the Feynman gauge or the Landau gauge.)

Belinfante's argument starts with the general expression of the gauge-invariant photon field given as

$$
\tilde{A}_{\mu}(x)=\int_{-\infty}^{0} F_{v \sigma}(z) \frac{\partial z^{v}}{\partial x^{\mu}} \frac{\partial z^{\sigma}}{\partial \xi} d \xi
$$

where $z^{v}(x, \xi)$ represents a point on the line toward $x$, with $\xi$ being a parameter chosen to satisfy the following boundary condition :

$$
z^{v}(x, 0)=x^{v} \text { and } z^{v}(x,-\infty)=\text { spatial infinity. }
$$

Belinfante first chose particularly simple paths at a fixed Lorentz frame. They are straight line paths at constant time, converging toward the field points $\boldsymbol{x}$ in arbitrary direction specified by unit vector $\epsilon$ :

$$
z^{v}(x, \xi)=x^{v}+\epsilon^{v} \xi
$$


where one can set without loss of generality as

$$
\epsilon^{0}=0, \quad \boldsymbol{\epsilon}^{2}=1, \quad \xi=-r \equiv|z-\boldsymbol{x}| .
$$

With this choice of path, Eq. (188) reduces to

$$
\tilde{A}_{\mu}(x)=\int_{-\infty}^{0} F_{i \mu}(z) \epsilon^{i} d \xi
$$

This potential $\tilde{A}_{\mu}$ is by construction gauge-invariant but it depends on the direction of the unit vector $\epsilon$. To remove this directional dependence, Belinfante propose to average Eq. (192) over all possible direction of $\epsilon$ as

$$
\bar{A}_{\mu}(x) \equiv \int \tilde{A}_{\mu}(x) \frac{d \Omega}{4 \pi},
$$

where $d \Omega$ is the infinitesimal solid angle in the direction of $\epsilon$. By using the identities

$$
d^{3} z=r^{2} d r d \Omega
$$

and

$$
|d \xi|=|d r|=\frac{d^{3} z}{r^{2}} d \Omega
$$

it can be shown that $\bar{A}_{\mu}(x)$ reduces to the following form :

$$
\bar{A}_{\mu}(x)=\int d^{3} z F_{i \mu}(z) \frac{\partial}{\partial z_{i}}\left(\frac{1}{4 \pi r}\right)=-\int d^{3} z\left[\nabla^{i} F_{i \mu}(z)\right]\left(\frac{1}{4 \pi r}\right) .
$$

If expressed with components, this reads as

$$
\bar{A}^{0}(x)=\int d^{3} z \frac{\nabla \cdot \boldsymbol{E}(z, t)}{4 \pi r}, \quad \overline{\boldsymbol{A}}(x)=\int d^{3} z \frac{\nabla \times \boldsymbol{B}(z, t)}{4 \pi r} .
$$

One therefore finds that the resultant potential $\bar{A}_{\mu}(x)$ just coincides with the vector potential in the Coulomb gauge. Thus, by averaging over the direction of straight-line paths, one could remove the line-dependence from the original DeWitt gauge potential. Besides, since it is expressed with the electric and magnetic fields only, it is obviously gauge-invariant. This observation lets us reconfirm the one and only nature of the Coulomb gauge among plural gauge choices. However, an attention must be paid to the the following facts. First, for that the above statement is meaningful, the integrals in Eq. 197) must converge. The condition of convergence is just the same as the condition of the Helmholtz theorem to hold. Second, the above path averaging is carried out over the direction of the straight lines at the constant time in a fixed Lorentz frame. This restriction is nothing serious as far as we are dealing with the electrodynamics of non-relativistic charged particles in a prescribed laboratory frame. This is the reason why the Coulomb gauge is the most convenient choice for such problems. As discussed in [29], however, the situation changes drastically for fully relativistic problems like the nucleon spin decomposition problem.

In the nucleon spin decomposition problem, two new features enter into the game. First, the nucleon is a strongly-coupled bound state of quarks and gluons described by a nonabelian gauge theory called the quantum chromodynamics. Second, the only way to empirically verify the proposed nucleon spin decomposition is to use deep-inelastic-scattering (DIS) measurements, in 
which the relativity plays crucial roles. As to the first point, it is known that the idea of gaugeinvariant (but path-dependent) electron and photon field can be extended to the nonabelian gauge theory with a slight modification [30],[31]. (The application of the gauge-invariant formulation of the nonabelian gauge theory to the nucleon spin decomposition problem was discussed in [32],[33],[34].) More important here is the second point, i.e. the proper treatment of the relativistic kinematics [29]. Within the relativistic framework, the path for defining the gauge-invariant gluon and quark fields can generally be chosen in 4-dimensional space-time manifold. Here, instead of discussing the general path choices in the gauge-invariant formulation of nonabelian gauge theory, we confine to several simple but important choices of path in the Minkowski space, which is widely believed to be equivalent to working in the so-called physical gauges.

The most popular physical gauges are the temporal gauge $\left(A^{0}=0\right)$, the light-cone gauge $\left(A^{+}=0\right.$ with $\left.A^{+} \equiv\left(A^{0}+A^{3}\right) / \sqrt{2}\right)$, the spatial axial gauge $\left(A^{3}=0\right)$, and the Coulomb gauge $(\nabla \cdot \boldsymbol{A}=0)$. We first recall the fact that selecting the physical component of the gauge field is intimately connected with choosing a particular gauge. In the general axial gauges, the physical component (or the gauge-covariant part) of the gluon field is known to be expressed as

$$
A_{\text {phys }}^{\mu}(x)=n_{v} \int_{-\infty}^{\infty} d \lambda(\mp \theta( \pm \lambda)) \mathcal{L}[x ; x+n \lambda] F^{\mu v}(x+n \lambda) \mathcal{L}[x+n \lambda ; x] .
$$

Here, $F^{\mu v}$ is the field strength tensor of the gluon field, while

$$
\mathcal{L}[y ; x]=\mathcal{P} \exp \left(i g \int_{x}^{y} d z_{\mu} A^{\mu}(z)\right),
$$

is a gauge-link (also called the Wilson line) connecting the two space-time points $x$ and $y$ with a straight line, $\mathcal{P}$ is the path-ordering operator, whereas $n^{\mu}$ is a constant four-vector characterizing the direction of the path. The dependence on the direction of $n^{\mu}$ is generally believed to reflect the gauge-dependence of the idea of the physical component of the gauge field. The familiar three physical gauges correspond to the following choice of $n^{\mu}$ :

$$
n^{\mu}=\left\{\begin{array}{lll}
(1,0,0,0) & \Leftrightarrow & \text { temporal gauge, } \\
\frac{1}{\sqrt{2}}(1,0,0,-1) & \Leftrightarrow & \text { light-cone gauge, } \\
(0,0,0,1) & \Leftrightarrow & \text { spatial axial gauge. }
\end{array}\right.
$$

In fact, with these choices of $n^{\mu}$, the physical component of the gluon field defined by (198) satisfies the constraint,

$$
n_{\mu} A_{\text {phys }}^{\mu}=0
$$

which is nothing but the gauge-fixing conditions of the general axial gauges. Since there is no particular spatial direction in the Coulomb gauge-fixing condition, the corresponding physical component may be obtained by averaging over path-dependent potential over the direction of all straight line paths at constant time, as in the abelian case.

Confining here to the three straight-line paths corresponding to the general axial gauges, it can be readily verified that any choice of $n^{\mu}$ gives the physical component of the gluon field, which transforms gauge-covariantly, i.e. as

$$
A_{p h y s}^{\mu}(x) \rightarrow U(x) A_{p h y s}^{\mu}(x) U^{-1}(x),
$$

under an arbitrary gauge transformations $U(x)=e^{i \omega_{a}(x) T_{a}}$. This enables us to write down gaugeinvariant expression of the gluon spin as well as that of the g.i.c. OAM of quarks in the nucleon 
[35],[36]. (More precisely, the gluon spin should be called the gluon helicity or the longitudinal component of the gluon spin with respect to the direction of the nucleon momentum.) They are given as

$$
\Delta G=\frac{1}{2 p^{+}}\left\langle p, s\left|2 \operatorname{Tr}\left[\epsilon_{\perp}^{j k} F^{j+}(0) A_{p h y s}^{k}(0)\right]\right| p, s\right\rangle
$$

and

$$
L_{z}^{\text {g.i.c. }}=\frac{\mathcal{N}^{\prime}}{2 p^{+}}\left\langle p, s\left|\int d^{2} r_{\perp} \bar{\psi}\left(0, \boldsymbol{r}_{\perp}\right) \gamma^{+} \frac{1}{i}\left(\boldsymbol{r}_{\perp} \times \boldsymbol{D}_{\text {pure }}\right)_{z} \psi\left(0, \boldsymbol{r}_{\perp}\right)\right| p, s\right\rangle,
$$

where $\mathcal{N}^{\prime}=1 /\left(\int d^{2} r_{\perp}\right), \epsilon_{\perp}^{j k}(j, k=1,2)$ is the antisymmetric tensor in two spatial dimension, and $|p, s\rangle$ is the nucleon state with momentum $p$ and spin $s$. The pure-gauge covariant derivative is defined by $\boldsymbol{D}_{\text {pure }} \equiv \nabla-i g \boldsymbol{A}_{\text {pure }}$ with $\boldsymbol{A}_{\text {pure }} \equiv \boldsymbol{A}-\boldsymbol{A}_{\text {phys }}$. Although the above quantities are formally gauge-invariant, they are path-dependent, just because they contains the quantity $\boldsymbol{A}_{\text {phys }}$, whose definition is path-dependent and non-local. It should be contrasted with the expression of the mechanical OAM of quarks given as

$$
L_{z}^{\text {g.i.c. }}=\frac{\mathcal{N}}{2 p^{+}}\left\langle p, s\left|\int d^{3} r \bar{\psi}(\boldsymbol{r}) \gamma^{+} \frac{1}{i}(\boldsymbol{r} \times \boldsymbol{D})_{z} \psi(\boldsymbol{r})\right| p, s\right\rangle,
$$

where $\mathcal{N}=1 /\left(\int d^{3} r\right)$, while $\boldsymbol{D}=\nabla-i g \boldsymbol{A}$ is the standard covariant derivative. Since the standard covariant derivative contains the full gauge field, the definition of the mechanical OAM is gauge-invariant as well as path-independent. Also interesting to remember is the relation between the g.i.c. OAM and the mechanical OAM. Using the definition $\boldsymbol{A}_{\text {pure }}=\boldsymbol{A}-\boldsymbol{A}_{\text {phys }}$, one can readily verify the following relation

$$
L_{z}^{\text {g.i.c. }}=L_{z}^{\text {mech }}+L_{z}^{\text {pot }}
$$

where

$$
L_{z}^{p o t}=\frac{\mathcal{N}^{\prime}}{2 p^{+}}\left\langle p, s\left|\int d^{2} r_{\perp} \bar{\psi}\left(0, \boldsymbol{r}_{\perp}\right) \gamma^{+} g\left(\boldsymbol{r}_{\perp} \times \boldsymbol{A}_{p h y}\right)_{z} \psi\left(0, \boldsymbol{r}_{\perp}\right)\right| p, s\right\rangle,
$$

is nothing but the potential angular momentum in the terminology of the papers [4], [5]. One can then say that the path-dependence of the g.i.c. OAM originates from that of the potential angular momentum, which explicitly contains the path-dependent (or direction-dependent) quantity $\boldsymbol{A}_{\text {phys. }}$.

In any case, it would be true that the definition of the gluon spin as well as that of the g.i.c. OAM are (at least formally) gauge-invariant irrespectively of the choice of $n^{\mu}$. This means that, from the viewpoint of gauge symmetry alone, these three choices of $n^{\mu}$ is totally on an equal footing, and anyone is neither superior nor inferior to the others. However, an important lesson leaned from our analysis of the Landau problem is a clear understanding about the meaning and the consequence of the path choice. Namely, within the gauge-invariant but path-dependent formulation of gauge theory a la DeWitt, what results from a particular choice of path is a particular symmetry of the quantum system or its wave function. We emphasize that this symmetry has nothing to do with the gauge symmetry. In fact, remember that, in the Landau problem, the choice of the path $C_{1}$ in the rectangular coordinate amounts to respecting the translational symmetry with respect to the $x$-axis. On the other hand, if we take the path $C_{I}$ in the cylindrical coordinate, it amounts to respecting the axial symmetry or the rotational symmetry around the $z$-axis. 
The understanding above obtained from the Landau problem gives a deep insight into our discussion of the nucleon spin decomposition problem. The question here is what symmetry is respected by a particular choice of the path in the 4-dimensional Minkowski space. Undoubtedly, if we choose the straight-line path along the $z$-direction, what is respected is the rotational symmetry around the $z$-axis. On the other hand, if we choose the straight-line path along the light-cone direction, an additional symmetry is respected besides the above two-dimensional rotation symmetry. It is the Lorentz boost-invariance with respect to the $z$-axis, which is the momentum direction of the parent nucleon. (For the detail, see [29].) What symmetry is respected if we choose the straight-line path along the time direction, then ? Since there is no particular spatial direction for this choice, this choice of path respects the full rotational symmetry in the 3-dimensional space. (This is also expected from the known intimate connection between the temporal gauge and the Coulomb gauge. Remember that, within the framework of the gauge-invariant formulation of DeWitt, the Coulomb gauge is obtained by averaging over the directions of straight-line paths in constant-time plane, so that there is no particular direction in three-dimensional space.)

Why is the boost-invariance along the z-direction so important? The reason is because the DIS measurement is the only way to experimentally verify the proposed nucleon spin decomposition, and that the DIS amplitude has a light-cone dominance [29]. In the inclusive or semiinclusive DIS processes, the ejected parton (a quark or a gluon) after being struck by high-energy virtual photon travels along the light cone with the speed of light. The light-like gauge link with the choice $n^{\mu}=\frac{1}{\sqrt{2}}(1,0,0,-1)$, which is also contained in the definition of $A_{\text {phys }}^{\mu}$, is known to simulate the interaction between the ejected parton and the residual target in the DIS processes. Very importantly, the gluon spin (or the g.i.c OAM of quarks) defined by the physical component of the gluon with the light-like gauge link has an important property, which must be satisfied by any PDFs [37]. That is the Lorentz-boost invariance along the direction of the parent nucleon. Putting it in another way, the physics favors the path in the light-cone direction in our nucleon spin decomposition problem [35].

As a last comment, since there is a definite physical meaning in the choice of path or the direction for defining the physical component of the gluon field, not only the mechanical OAM but also the canonical OAM are thought to have some definite physical meaning [29], [38]. What these two OAMs really mean in the nucleon spin decomposition problem was already discussed in a recent paper [15]. It was shown there that what represents the OAM of quarks inside the nucleon is the mechanical OAM not the canonical OAM. The latter represents the OAM of a quark in the asymptotic distance (well outside the nucleon) after leaving the target nucleon in the semi-inclusive DIS scattering processes. In this sense, it would be legitimate to say that the canonical type decomposition of the nucleon spin, also called the Jaffe-Manohar decomposition of the nucleon spin [39], [40], is not such a decomposition that genuinely represents the intrinsic (or static) property of the nucleon .

\section{Summary and concluding remarks}

The delicacy of the gauge choice in the Landau problem partially stems from a singular nature of the magnetic field, which uniformly spread over the whole plane. In fact, if the magnetic field is confined in a finite domain, the vector potential, or more precisely its transverse component, is essentially uniquely fixed by the Helmholtz theorem. As was explicitly shown in the case of infinitely long solenoid with finite cross-sectional area, what emerges here is just the gauge 
potential of symmetric gauge or the Coulomb gauge. (To be more precise, in the two dimensional problem, there still remains a freedom to choose the Bawin-Burnel type gauge, although it is a singular and multi-valued gauge.) The uniform magnetic field in the Landau problem allows entirely different gauge from the symmetric gauge, namely, the two types of Landau gauge. The choice of the Landau gauges favors the rectangular coordinate treatment of the problem, while the choice of the symmetric gauge does the cylindrical coordinate treatment, so that they lead to the eigen-functions with totally different appearances. It turns out that, because of the degeneracy of the Landau levels in both gauges, the eigen-functions in both gauges are not connected by a simple gauge transformation. Rather, what are related to the eigen-functions in the symmetric gauge by the gauge transformation is a particular linear combination of the eigen-functions in the Landau gauge. We showed that the weight function of this linear combination is nothing but the matrix element of the (unitary) gauge transformation operator sandwiched with the eigen-states of the Landau gauge and those of the symmetric gauge. We also derived the explicit form of this weight function.

In addition to the above-mentioned standard formulation of the Landau problem, we have also investigated the same problem on the basis of the gauge-invariant but path-dependent formulation of the quantum electrodynamics $a$ la DeWitt. A great advantage of this gauge-invariant formulation is that, with the help of the path-dependent (non-local) phase factor, we can solve the Schrödinger equation without specifying explicit form of the gauge potential. With the choice of the two polygonal line paths in the rectangular coordinate, we are led to the eigen-functions expressed by the Hermite polynomials except for a nonlocal phase factor. It was shown that these choices of path have a close connection with the two types of Landau gauge. On the other hand, with the choice of straight-line path in the cylinder coordinate, we are led to the eigen-functions expressed by the associated Laguerre polynomial, again except for nonlocal phase factors. This choice of path then has an intimate connection with symmetric gauge choice. Still, a highly nontrivial fact is that these latter eigen-functions are given for arbitrary form of the vector potential, which especially allows us to take the vector potential in either of the two Landau gauges. In this Landau gauge limit, however, the above eigen-functions do not reduce to the standard eigen-functions in the Landau gauge. Rather, they reduce to a particular linear combination of the eigen-functions in the Landau gauge, which is the same object already obtained in our discussion of the standard (gauge-fixed) formulation of the Landau problem.

Through the study of DeWitt's gauge-invariant formulation of the Landau problem, we realize that the choice of path has an intimate connection with the choice of gauge. For instance, the choice of a polygonal line path $C_{1}$ in the rectangular coordinate system is shown to have inseparable connection with the choice of the 1 st Landau gauge, whereas the choice of the path $C_{I}$ in the cylindrical coordinate has a close connection with the choice of symmetric gauge. Nonetheless, highly nontrivial fact is that the choice of path is not absolutely equivalent to the choice of gauge, because we still have freedom to take any gauges for each choice of path. A question is therefore what is meant by the path choice in the gauge-invariant but path-dependent formulation of DeWitt. We conclude that the choice of path in DeWitt's formalism is equivalent to selecting a particular symmetry in our quantum mechanical problem. As we have shown, the choice of the polygonal line path $C_{1}$ amounts to respecting the translational symmetry with respect to the $x$-axis. Once the gauge-invariant wave functions are constructed so as to satisfy this symmetry, the expectation value of the g.i.c. momentum operator $K_{x}$ is shown to be independent of the three choices of gauges, i.e. the two Landaus and the symmetric gauge at variance with that of the ordinary canonical momentum operator $p_{x}$. Nevertheless, this gauge-independent nature of the g.i.c. momentum should be taken with care. In fact, we compare the expectation value of the 
g.i.c. momentum and that of the mechanical momentum, thereby confirming the physical nature of the latter as compared with the former. On the other hand, if we choose the path $C_{I}$ in the cylindrical coordinate, it amounts to respecting the rotational symmetry around the $z$-axis. Once the gauge-invariant wave functions are constructed so as to meet this symmetry, we find that the expectation value the g.i.c. OAM and that of the standard canonical OAM are both independent of the three choices of gauge and they just coincide. This is thought to be a consequence of the rotational symmetry around the $z$-axis. However, the expectation value of these two types of canonical OAM is different from that of the mechanical OAM. The physical nature of the mechanical OAM (as compared with the others) is thought to manifest in its relation with the observable Landau energies.

We have also discussed the issue of gauge choice in the nucleon spin decomposition problem from the general viewpoint of the gauge-invariant but path-dependent formulation of nonabelian gauge theory. As we have argued, for relativistic problems like the nucleon spin decomposition problem, the path for defining the physical component of the gauge field can be chosen arbitrarily in the 4-dimensional Minkowski space. Among several simple choices of paths, i.e. the straight line in the time direction, the light-cone direction, and the $z$-direction, only the light-cone path choice can satisfy the required symmetries of the physics in question, i.e. the rotational symmetry around the $z$-axis as well as the Lorentz-boost invariance along the same direction. Accordingly, only the gluon spin operator (or g.i.c OAM of quarks) defined by the physical component of the gluon with the light-like gauge link can satisfy the Lorentz-boost invariance along the momentum direction of the parent nucleon, which must be respected by any parton distribution functions. In other words, what selects the proper definition of the physical component of the gluon field in the nucleon spin decomposition problem is the Lorentz symmetry rather than the gauge symmetry [29]. We recall that the importance of Lorentz symmetry in the proper definition of the physical component of the gluon field was already stressed by Tiwari at the early stage of debate [41].

\section{Acknowledgement}

M.W. would like to thank S.C. Tiwari for several useful comments. M.W. also thanks the Institute of Modern Physics of the Chinese Academy of Sciences in Lanzhou for hospitality. Y.K. and P.M.Z. are supported by the National Natural Science Foundation of China (Grant No.11575254). This work is partly supported by the Chinese Academy of Sciences President's International Fellowship Initiative (No. 2018VMA0030 and No. 2018PM0028)

\section{Appendix A. Calculation of gauge transformation matrix}

In this appendix, we evaluate the matrix element of the gauge transformation operator between the eigenstates in the Landau gauge and those in the symmetric gauge. Here, we consider the $m \geq 0$ case, since the $m<0$ case can be handled similarly,

$$
\begin{gathered}
\left\langle\Psi_{n^{\prime}, k_{y}}^{(L)}\left|e^{-i \frac{1}{2} e B x y}\right| \Psi_{n, m}^{(S)}\right\rangle=\int d x d y \frac{1}{\sqrt{2 \pi}} e^{-i k_{y} y} N_{n^{\prime}} H_{n^{\prime}}\left(\frac{x-x_{0}}{l_{B}}\right) e^{-\frac{\left(x-x_{0}\right)^{2}}{2 l_{B}^{2}}} \\
\times e^{-i \frac{1}{2} e B x y} \frac{1}{\sqrt{2 \pi}} N_{n, m} e^{i m \phi}\left(\frac{r^{2}}{2 l_{B}^{2}}\right)^{m / 2} e^{-\frac{r^{2}}{4 l_{B}^{2}}} L_{n-m}^{m}\left(\frac{r^{2}}{2 l_{B}^{2}}\right) .
\end{gathered}
$$


Using the notation $z=x+i y=r e^{i \phi}$ and $\bar{z}=x-i y$, we rewrite it as

$$
\begin{aligned}
\left\langle\Psi_{n^{\prime}, k_{y}}^{(L)}\left|e^{-i \frac{1}{2} e B x y}\right| \Psi_{n, m}^{(S)}\right\rangle & =\frac{1}{2 \pi} \int d x d y e^{-i k_{y} y} N_{n^{\prime}} H_{n^{\prime}}\left(\frac{x-x_{0}}{l_{B}}\right) e^{-\frac{\left(x-x_{0}\right)^{2}}{2 l_{B}^{2}}} \\
\times e^{-i \frac{1}{2} e B x y} & \times N_{n, m} 2^{m / 2}\left(\frac{z}{2 l_{B}}\right)^{m} e^{-\frac{\bar{z} z}{4 l_{B}^{2}}} L_{n-m}^{m}\left(\frac{\bar{z} z}{2 l_{B}^{2}}\right) .
\end{aligned}
$$

Now, with use of the mathematical identity

$$
\int_{-\infty}^{\infty} d u e^{-u^{2}} H_{p}(u+v) H_{q}(u+w)=\sqrt{\pi} 2^{q} p ! w^{q-p} L_{p}^{q-p}(-2 v w) \quad(\text { for } p \leq q),
$$

we can write as

$$
\left(\frac{z}{2 l_{B}}\right)^{m} L_{n-m}^{m}\left(\frac{\bar{z} z}{2 l_{B}^{2}}\right)=\frac{(-1)^{m}}{\sqrt{\pi} 2^{n}(n-m) !} \int_{-\infty}^{\infty} d u e^{-u^{2}} H_{n-m}\left(u+\frac{\bar{z}}{2 l_{B}}\right) H_{n}\left(u-\frac{z}{2 l_{B}}\right) .
$$

Thus we find that

$$
\begin{aligned}
\left\langle\Psi_{n^{\prime}, k_{y}}^{(L)}\left|e^{-i \frac{1}{2} e B x y}\right| \Psi_{n, m}^{(S)}\right\rangle & =\frac{1}{2 \pi} \int d x d y e^{-i k_{y} y} N_{n^{\prime}} H_{n^{\prime}}\left(\frac{x-x_{0}}{l_{B}}\right) e^{-\frac{\left(x-x_{0}\right)^{2}}{2 l_{B}^{2}}} \\
& \times e^{-i \frac{1}{2} e B x y} \times N_{n, m} 2^{m / 2} \frac{(-1)^{m}}{\sqrt{\pi} 2^{n}(n-m) !} e^{-\frac{\bar{z} z}{4 l_{B}^{2}}} \\
& \times \int_{-\infty}^{\infty} d u e^{-u^{2}} H_{n-m}\left(u+\frac{\bar{z}}{2 l_{B}}\right) H_{n}\left(u-\frac{z}{2 l_{B}}\right)
\end{aligned}
$$

To proceed, first we note that

$$
e^{-i k_{y} y} e^{-i \frac{1}{2} e B x y}=e^{-i \frac{1}{2 l_{B}^{2}}\left(x-2 x_{0}\right) y} .
$$

Next, we introduce a variable transformation from $u$ to $w=u-i y /\left(2 l_{B}\right)$, which gives

$$
\begin{aligned}
& u+\frac{\bar{z}}{2 l_{B}}=u-\frac{i y}{2 l_{B}}+\frac{x}{2 l_{B}}=w+\frac{x}{2 l_{B}}, \\
& u-\frac{z}{2 l_{B}}=u-\frac{i y}{2 l_{B}}-\frac{x}{2 l_{B}}=w-\frac{x}{2 l_{B}} .
\end{aligned}
$$

Now, using the manipulations

$$
\begin{aligned}
& e^{-u^{2}}=e^{-\left(w+\frac{i y}{2 l_{B}}\right)^{2}}=e^{-w^{2}} e^{-i \frac{w y}{l_{B}}} e^{\frac{y^{2}}{4 l_{B}^{2}}}, \\
& e^{-\frac{\left(x-x_{0}\right)^{2}}{2 l_{B}^{2}}} e^{-\frac{\bar{z} z}{4 l_{B}^{2}}} e^{-u^{2}}=e^{-\frac{\left(x-x_{0}\right)^{2}}{2 l_{B}^{2}}} e^{-\frac{x^{2}}{4 l_{B}^{2}}} e^{-w^{2}} e^{-i \frac{w y}{l_{B}}},
\end{aligned}
$$

as well as

$$
e^{-i \frac{1}{2 l_{B}^{2}}\left(x-2 x_{0}\right) y} e^{-i \frac{w y}{l_{B}}}=e^{-i \frac{1}{T_{B}}\left(w+\frac{x-2 x_{0}}{2 l_{B}}\right) y},
$$


we obtain

$$
\begin{aligned}
\left\langle\Psi_{n^{\prime}, k_{y}}^{(L)}\left|e^{-\frac{1}{2} i e B x y}\right| \Psi_{n, m}^{(S)}\right\rangle & =\frac{1}{2 \pi} N_{n^{\prime}} N_{n, m} \frac{(-1)^{m} 2^{m / 2}}{\sqrt{\pi} 2^{n}(n-m) !} \int d x d y \int d w H_{n^{\prime}}\left(\frac{x-x_{0}}{l_{B}}\right) \\
& \times e^{-i \frac{1}{l_{B}}\left(w+\frac{x-2 x_{0}}{2 l_{B}}\right) y} e^{-\frac{\left(x-x_{0}\right)^{2}}{2 l_{B}^{2}}} e^{-\frac{x^{2}}{4 l_{B}^{2}}} e^{-w^{2}} \\
& \times H_{n-m}\left(u+\frac{\bar{z}}{2 l_{B}}\right) H_{n}\left(u-\frac{z}{2 l_{B}}\right) .
\end{aligned}
$$

The integration over the variable $w$ can readily be done as

$$
\int_{-\infty}^{\infty} d y e^{-i \frac{1}{\tau_{B}}\left(w+\frac{x-2 x_{0}}{2 l_{B}}\right) y}=2 \pi l_{B} \delta\left(w+\frac{x-2 x_{0}}{2 l_{B}}\right),
$$

which makes the integration over $w$ trivial, thereby leading to the result :

$$
\begin{aligned}
\left\langle\Psi_{n^{\prime}, k_{y}}^{(L)}\left|e^{-\frac{1}{2} i e B x y}\right| \Psi_{n, m}^{(S)}\right\rangle & =\frac{1}{2 \pi} N_{n^{\prime}} N_{n, m} l_{B} \frac{(-1)^{m} 2^{m / 2}}{\sqrt{\pi} 2^{n}(n-m) !} \int d x H_{n^{\prime}}\left(\frac{x-x_{0}}{l_{B}}\right) \\
& \times e^{-\frac{\left(x-x_{0}\right)^{2}}{2 l_{B}^{2}}} e^{-\frac{x^{2}}{4 l_{B}^{2}}} e^{-\frac{\left(x-2 x_{0}\right)^{2}}{4 l_{B}^{2}}} H_{n-m}\left(\frac{x_{0}}{l_{B}}\right) H_{n}\left(-\frac{x-x_{0}}{l_{B}}\right) .
\end{aligned}
$$

Using the relation

$$
e^{-\frac{\left(x-x_{0}\right)^{2}}{2 l_{B}^{2}}} e^{-\frac{x^{2}}{4 l_{B}^{2}}} e^{-\frac{\left(x-2 x_{0}\right)^{2}}{4 l_{B}^{2}}}=e^{-\frac{\left(x-x_{0}\right)^{2}}{l_{B}^{2}}} e^{-\frac{x_{0}^{2}}{2 l_{B}^{2}}},
$$

and the identity $H_{n}(-x)=(-1)^{n} H_{n}(x)$ of the Hermite polynomial, we therefore get

$$
\begin{aligned}
\left\langle\Psi_{n^{\prime}, k_{y}}^{(L)}\left|e^{-i \frac{1}{2} e B x y}\right| \Psi_{n, m}^{(S)}\right\rangle & =\frac{1}{2 \pi} N_{n^{\prime}} N_{n, m} l_{B} \frac{(-1)^{n+m} 2^{m / 2}}{\sqrt{\pi} 2^{n}(n-m) !} H_{n-m}\left(\frac{x_{0}}{l_{B}}\right) e^{-\frac{x_{0}^{2}}{2 l_{B}^{2}}} \\
& \times \int d x e^{-\frac{\left(x-x_{0}\right)^{2}}{l_{B}^{2}}} H_{n^{\prime}}\left(\frac{x-x_{0}}{l_{B}}\right) H_{n}\left(\frac{x-x_{0}}{l_{B}}\right) .
\end{aligned}
$$

Now, by using the familiar ortho-normalization relation of the Hermite polynomial as well as the definitions of the normalization constants $N_{n}$ and $N_{n, m}$, we arrive at the desired relation,

$$
\left\langle\Psi_{n^{\prime}, k_{y}}^{(L)}\left|e^{-i \frac{1}{2} e B x y}\right| \Psi_{n, m}^{(S)}\right\rangle=\delta_{n^{\prime}, n} U_{n, m}\left(x_{0}\right),
$$

where

$$
U_{n, m}\left(x_{0}\right)=C_{n, m} H_{n-m}\left(\frac{x_{0}}{l_{B}}\right) e^{-\frac{x_{0}^{2}}{2 l_{B}^{2}}}
$$

with

$$
C_{n, m}=l_{B}\left(\frac{1}{\sqrt{\pi} 2^{n-m}(n-m) ! l_{B}}\right)^{1 / 2}
$$




\section{Appendix B. Evaluation of the weighted integral}

Here we show the calculation of the weighted integral $\mathcal{I}$ in Eq.(42),

$$
\mathcal{I}=\int d k_{y} U_{n, m}\left(x_{0}\right) \Psi_{n, k_{y}}^{(L)}(x, y) .
$$

We again assume $m \geq 0$, since the $m<0$ case can be treated entirely in the same way. Inserting the expressions of $U_{m, n}\left(x_{0}\right)$ and $\Psi_{n, k_{y}}^{(L)}(x, y)$ into the above equation, and using the relation $x_{0}=$ $-l_{B}^{2} k_{y}$, we have

$$
\mathcal{I}=\frac{1}{l_{B}^{2}} C_{n, m} \int d x_{0} H_{n-m}\left(\frac{x_{0}}{l_{B}}\right) e^{-\frac{x_{0}^{2}}{2 l_{B}^{2}}} \frac{1}{\sqrt{2 \pi}} N_{n} e^{i k_{y} y} H_{n}\left(\frac{x-x_{0}}{l_{B}}\right) e^{-\frac{\left(x-x_{0}\right)^{2}}{2 l_{B}^{2}}} .
$$

Using the equality

$$
e^{-\frac{x_{0}^{2}}{2 l_{B}^{2}}} e^{i k_{y} y} e^{-\frac{\left(x-x_{0}\right)^{2}}{2 l_{B}^{2}}}=e^{-\frac{1}{l_{B}^{2}\left(x_{0}-\frac{\xi}{2}\right)^{2}}} e^{-\frac{1}{4 l_{B}^{2}} \bar{z} z} e^{-i \frac{1}{2} e B x y},
$$

we thus obtain

$$
\begin{aligned}
\mathcal{I}= & \frac{1}{l_{B}^{2}} C_{n, m} \frac{1}{\sqrt{2 \pi}} N_{n} e^{-\frac{1}{4 l_{B}^{2}} \bar{z} z} e^{-i \frac{1}{2} e B x y} \\
& \times \int d x_{0} e^{-\frac{1}{l_{B}^{2}}\left(x_{0}-\frac{\bar{z}}{2}\right)^{2}} H_{n-m}\left(\frac{x_{0}}{l_{B}}\right) H_{n}\left(\frac{x-x_{0}}{l_{B}}\right) .
\end{aligned}
$$

After the variable transformation from $x_{0}$ to $u$ with

$$
x_{0}-\frac{\bar{z}}{2}=l_{B} u,
$$

the above expression can be transformed into the form,

$$
\begin{aligned}
I= & \frac{1}{l_{B}}(-1)^{n} C_{n, m} \frac{1}{\sqrt{2 \pi}} N_{n} e^{-\frac{1}{4 l_{B}^{2}} \bar{z} z} e^{-i \frac{1}{2} e B x y} \\
& \times \int d u e^{-u^{2}} H_{n-m}\left(u+\frac{\bar{z}}{2 l_{B}}\right) H_{n}\left(u-\frac{z}{2 l_{B}}\right) .
\end{aligned}
$$

Using the identity $(\mathrm{A.3}$, we therefore get

$$
\begin{aligned}
& \int d u e^{-u^{2}} H_{n-m}\left(u+\frac{\bar{z}}{2 l_{B}}\right) H_{n}\left(u-\frac{z}{2 l_{B}}\right) \\
& =(-1)^{m} 2^{n} \sqrt{\pi}(n-m) !\left(\frac{z}{2 l_{B}}\right)^{m} L_{n-m}^{m}\left(\frac{\bar{z} z}{2 l_{B}^{2}}\right) .
\end{aligned}
$$

This gives

$$
\begin{aligned}
I= & \frac{1}{l_{B}}(-1)^{n} C_{n, m} \frac{1}{\sqrt{2 \pi}} N_{n} e^{-\frac{1}{4 l_{B}^{2}} \bar{z} z} e^{-i \frac{1}{2} e B x y} \\
& \times(-1)^{m} 2^{n} \sqrt{\pi}(n-m) !\left(\frac{z}{2 l_{B}}\right)^{m} L_{n-m}^{m}\left(\frac{\bar{z} z}{2 l_{B}^{2}}\right) .
\end{aligned}
$$


Finally, with use of the definitions of the coefficients $C_{n, m}$ and $N_{n}$, we find that

$$
\mathcal{I} \equiv \int d k_{y} U_{n, m}\left(x_{0}\right) \Psi_{n, k_{y}}^{(L)}(x, y)=e^{-i \frac{1}{2} e B x y} \Psi_{n, m}^{(S)}(x, y) .
$$

\section{Appendix C. Proof of gauge-invariance of the electron wave function $\tilde{\Psi}^{\left(C_{\mathrm{I}}\right)}(x, y)$ given by} Eq.(115)

Here we prove that the electron wave function $\tilde{\Psi}^{\left(C_{\mathrm{I}}\right)}(x, y)$ defined by

$$
\tilde{\Psi}^{\left(C_{\mathrm{I}}\right)}(x, y) \equiv U_{I}^{-1} \Psi(x, y),
$$

with $U_{\mathrm{I}}=e^{-i e \int_{0}^{r} A_{r}\left(r^{\prime}, \phi\right) d r^{\prime}}$ is gauge-invariant. Under an arbitrary gauge transformation

$$
\begin{aligned}
\Psi(r, \phi) & \rightarrow e^{-i e[\omega(r, \phi)-\omega(0,0)]} \Psi(r, \phi), \\
\boldsymbol{A}(r \phi) & \rightarrow \boldsymbol{A}(r, \phi)+\nabla \omega(r, \phi),
\end{aligned}
$$

the radial and azimuthal components of $\boldsymbol{A}$ transforms as

$$
\begin{aligned}
& A_{r}(r, \phi) \rightarrow A_{r}(r, \phi)+\frac{\partial \omega(r, \phi)}{\partial r} \\
& A_{\phi}(r, \phi) \rightarrow A_{\phi}(r, \phi)+\frac{1}{r} \frac{\partial \omega(r, \phi)}{\partial \phi} .
\end{aligned}
$$

We first investigate the gauge-transformation property of the operator $U_{\mathrm{I}}^{-1}$. Only one delicate point here is that the line-integral contained in the definition of $U_{\mathrm{I}}^{-1}$ should be interpreted as $r_{0} \rightarrow 0$ limit of the following expression (see Fig C.3. ;

$$
U_{\mathrm{I}}^{-1}=\lim _{r_{0} \rightarrow 0} e^{i e\left[\int_{r_{0}}^{r} A_{r}\left(r^{\prime}, \phi\right) d r^{\prime}+\int_{0}^{\phi} r_{0} A_{\phi}\left(r_{0}, \phi^{\prime}\right) d \phi^{\prime}\right]} .
$$

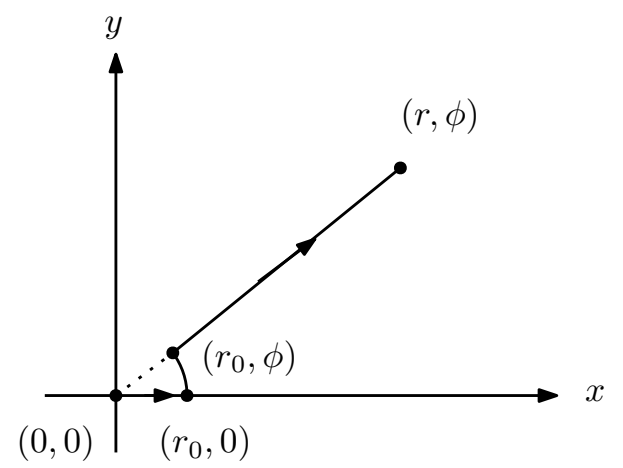

Figure C.3: The path $C_{\mathrm{I}}$ defined as $r_{0} \rightarrow 0$ limit of the path illustrated in this figure. 
Under the gauge transformation, the phase factor transforms as

$$
\begin{aligned}
& \int_{r_{0}}^{r} A_{r}\left(r^{\prime}, \phi\right) d r^{\prime}+\int_{0}^{\phi} r_{0} A_{\phi}\left(r_{0}, \phi^{\prime}\right) d \phi^{\prime} \\
\rightarrow & \int_{r_{0}}^{r}\left(A_{r}\left(r^{\prime}, \phi\right)+\frac{\partial}{\partial r^{\prime}} \omega\left(r^{\prime}, \phi\right)\right) d r \prime+\int_{0}^{\phi} r_{0}\left(A_{\phi}\left(r_{0} . \phi^{\prime}\right)+\frac{1}{r_{0}} \frac{\partial \omega\left(r_{0}, \phi^{\prime}\right)}{\partial \phi^{\prime}}\right) d \phi^{\prime} \\
= & \int_{r_{0}}^{r} A_{r}\left(r^{\prime}, \phi\right) d r^{\prime}+\int_{0}^{\phi} r_{0} A_{\phi}\left(r_{0}, \phi^{\prime}\right) d \phi^{\prime} \\
& +\left[\omega(r, \phi)-\omega\left(r_{0}, \phi\right)\right]+\left[\omega\left(r_{0}, \phi\right)+\omega(0,0)\right] \\
= & \int_{r_{0}}^{r} A_{r}\left(r^{\prime}, \phi\right) d r^{\prime}+\int_{0}^{\phi} r_{0} A_{\phi}\left(r_{0}, \phi^{\prime}\right) d \phi^{\prime}+[\omega(r, \phi)-\omega(0,0)] .
\end{aligned}
$$

In the limit $r_{0} \rightarrow 0$, this reduces to

$$
\int_{0}^{r} A_{r}\left(r^{\prime}, \phi\right) d r^{\prime}+[\omega(r, \phi)-\omega(0,0)]
$$

Thus, we find that, under the gauge transformation,

$$
\begin{aligned}
& U_{\mathrm{I}}^{-1} \rightarrow U_{\mathrm{I}}^{-1} e^{i e[\omega(r, \phi)-\omega(0,0)]}, \\
& \Psi(r, \phi) \rightarrow e^{-i e[\omega(r, \phi)-\omega(0,0)]} \Psi(r, \phi),
\end{aligned}
$$

and consequently

$$
\tilde{\Psi}^{\left(C_{\mathrm{I}}\right)}(r, \phi) \equiv U_{\mathrm{I}}^{-1} \Psi(r, \phi) \rightarrow \tilde{\Psi}^{\left(C_{\mathrm{I}}\right)}(r, \phi),
$$

which proves that $\tilde{\Psi}^{\left(C_{\mathrm{I}}\right)}(r, \phi)$ is gauge-invariant.

\section{Appendix D. The proof of the relation $\left\langle\Psi_{n, m}^{\left(C_{I}\right)}\left|L_{z}^{c a n}\right| \Psi_{n, m}^{\left(C_{I}\right)}\right\rangle=m$ for arbitrary gauge.}

With use of the expression (138) for $\Psi_{n, m}^{\left(C_{I}\right)}(r, \theta)$, we obtain

$$
\begin{aligned}
& \left\langle\Psi_{n, m}^{\left(C_{I}\right)}\left|L_{z}^{c a n}\right| \Psi_{n, m}^{\left(C_{I}\right)}\right\rangle=\left\langle\Psi_{n, m}^{\left(C_{I}\right)}\left|-i \frac{\partial}{\partial \phi}\right| \Psi_{n, m}^{\left(C_{l}\right)}\right\rangle \\
= & \int_{0}^{\infty} d r r \int_{0}^{2 \pi} d \phi R_{n, m}(r)\left(m+\frac{1}{2} e B r^{2}-e r A_{\phi}(r, \phi)\right) R_{n, m}(r) .
\end{aligned}
$$

Here, we make use of the relation

$$
r A_{\phi}(r, \phi)=\frac{1}{2} r^{2} B+\int_{0}^{r} d r^{\prime} \frac{\partial}{\partial \phi} A_{r}\left(r^{\prime}, \phi\right)
$$

which is obtained from the circular coordinate representation of the identity $B=(\nabla \times A)_{z}$. This gives

$$
\left\langle\Psi_{n, m}^{\left(C_{I}\right)}\left|L_{z}^{c a n}\right| \Psi_{n, m}^{\left(C_{I}\right)}\right\rangle=m-e \int_{0}^{\infty} d r r \int_{0}^{2 \pi} d \phi R_{n, m}(r) \int_{0}^{r} d r^{\prime} \frac{\partial}{\partial \phi} A_{r}\left(r^{\prime}, \phi\right) R_{n, m}(r) .
$$


Carrying out the integral over $\phi$ first, we find that

$$
\int_{0}^{2 \pi} d \phi \frac{\partial}{\partial \phi} A_{r}\left(r^{\prime}, \phi\right)=A_{r}\left(r^{\prime}, 2 \pi\right)-A_{r}\left(r^{\prime}, 0\right) .
$$

This vanishes, since it holds that

$$
A_{r}(r, \phi+2 \pi)=A_{r}(r, \phi), \quad A_{\phi}(r, \phi+2 \pi)=A_{\phi}(r, \phi),
$$

for arbitrary single-valued (or regular) gauge-field configuration. In this way, we obtain

$$
\left\langle\Psi_{n, m}^{\left(C_{l}\right)}\left|L_{z}^{c a n}\right| \Psi_{n, m}^{\left(C_{l}\right)}\right\rangle=m,
$$

for any single-valued gauge.

\section{References}

\section{References}

[1] E. Leader and C. Lorcé, Phys. Rep. 541 (2014) 163.

[2] M. Wakamatsu, Int. J. Mod. Phys. A 29 (2014) 1430012.

[3] K.-F. Liu and C. Lorcé, Eur. Phys. J. A52 (2016) 160.

[4] M. Wakamatsu, Phys. Rev. D81 (2010) 114010.

[5] M. Wakamatsu, Phys. Rev. D83 (2011) 014012.

[6] L.D. Landau, Z. Phys. 64 (1930) 629.

[7] L.D. Landau and E.M. Lifshitz, Quantum Mechanics : Non-Relativistic Theory, Course of Theoretical Physics, 3rd ed. (Pergamon, New York, 1977), Vol.3.

[8] G. Konstantinou and K. Moulopoulos, momentum, Eur. J. Phys. 37 (2016) 065401.

[9] G. Konstantinou and K. Moulopoulos, Int. J. Theor. Phys. 56 (2017) 1484.

[10] D. Yoshioka, The Quantum Hall Effects, (Springer, 2002), pp.20-23.

[11] X.-S. Chen, X.-F. Lü, W.-M. Sun, F.Wang, and T. Goldman, Phys. Rev. Lett. 100 (2008) 232002.

[12] X.-S. Chen,W.-M. Sun, X.-F. Lü, F.Wang, and T. Goldman, Phys. Rev. Lett. 103 (2009) 062001.

[13] H.-Y. Fan and J.-X. Lin, Phys. Lett. A267 (2000) 194.

[14] C.-F. Li and Q. Wang, Physica A (Amsterdam) B269 (1999) 22.

[15] M. Wakamatsu, Phys. Rev. D94 (2016) 056004.

[16] R.J. Swenson, Am. J. Phys. 57 (1989) 381.

[17] T. Haugset, J.Aa. Ruud, and F. Ravndal, Physica Scripta, 47 (1993) 715.

[18] B.S. DeWitt, Phys. Rev. 125 (1962) 2189.

[19] S. Mandelstam, Ann. Phys. (N.Y.) 19 (1962) 1.

[20] P.A.M. Dirac, Can. J. Phys. 33 (1955) 650.

[21] M. Bawin and A. Burnel J. Phys. A : Math. Gen. 16 (1983) 2173.

[22] K.-H. Yang, J. Phys. A 18 (1985) 979.

[23] C.R. Greenshields, R.L. Stamps, S. Franke-Arnold, and S.M. Barnett, Phys. Rev. Lett. 113 (2014) 240404.

[24] J.-F. Li, Y. Jiang, W.-M. Sun, H.-S.Zhong, and F. Wang, Mod. Phys. Lett. B26 (2012) 1250124.

[25] P. Schattschneider, Th. Schachinger, M. Stöger-Pollach, S.Löffler, A. Steiger-Thirsfeld, K.Y. Bliokh, and F. Nori, Nat. Commun. 5 (2014) 4586.

[26] K.Y. Bliokh, P. Schattschneider, J. Verbeeck, and F. Nori, Phys. Rev. X2 (2012) 041011.

[27] F. Belinfante, Phys. Rev. 128 (1962) 2832.

[28] F. Rohrlich and F. Strocchi, Phys. Rev. 139 (1965) B476.

[29] M. Wakamatsu, Eur. Phys. J. A51 (2015) 52.

[30] S.V. Ivanov and G.P. Korchemsky, Phys. Lett. 154B (1985) 197.

[31] S.V. Ivanov, G.P. Korchemsky, and A. Radyushkin, Yad. Fiz. 44 (1986) 230 [Sov. J. Nucl. Phys. 44 (1986) 145].

[32] M. Wakamatsu, Phys. Rev. D87 (2013) 094035. 
[33] C. Lorcé, Phys. Rev. D87 (2013) 034031.

[34] C. Lorcé, Phys. Rev. D88 (2013) 044037.

[35] Y. Hatta, Phys. Rev. D84 (2011) 041701.

[36] P.M. Zhang and D.G. Pak, Eur. Phys. J. A48 (2012) 91.

[37] J.C. Collins, Foundation of Perturbative QCD (Cambridge University Press, Cambridge, 2011).

[38] M. Burkardt, Phys. Rev. D88 (2013) 014014.

[39] R.L. Jaffe and A. Manohar, Nucl. Phys. B337 (1990) 509

[40] S.V. Bashinsky and R.L. Jaffe, Nucl. Phys. B536 (1998) 303.

[41] S.C. Tiwari, arXiv : 0807.0699 [physics.gen-ph] (2008). 